\title{
Magnetic-flux distribution and the magnetic penetration depth in superconducting polycrystalline $\mathrm{Bi}_{2} \mathrm{Sr}_{2} \mathrm{Ca}_{1-x} \mathrm{Y}_{x} \mathrm{Cu}_{2} \mathrm{O}_{8+\delta}$ and $\mathrm{Bi}_{2-x} \mathrm{~Pb}_{x} \mathrm{Sr}_{2} \mathrm{CaCu}_{2} \mathrm{O}_{8+\delta}$
}

\author{
M. Weber, A. Amato, F. N. Gygax, and A. Schenck \\ Institute for Intermediate Energy Physics, Swiss Federal Institute of Technology \\ (Eidgenössiche Technische Hochschule) Zürich, CH-5232 Villigen PSI, Switzerland \\ H. Maletta \\ Hahn-Meitner-Institut Berlin GmbH, D-W-1000 Berlin 39, Germany
}

\author{
V. N. Duginov, V. G. Grebinnik, A. B. Lazarev, V. G. Olshevsky, V. Yu. Pomjakushin, S. N. Shilov, \\ and V. A. Zhukov
}

Joint Institute for Nuclear Research, Dubna, Head Post Office, P.O. Box 79, Moscow, Russia

B. F. Kirillov, A. V. Pirogov, A. N. Ponomarev, and V. G. Storchak

Kurchatov Institute of Atomic Energy, 123182 Moscow, Russia

S. Kapusta

Institute of Physics, Czechoslovak Academy of Science, 25068 Rez, Czechoslovakia

J. Bock

Hoechst AG, Werk Knapsack, D-W-5030 Hürth, Germany

(Received 5 February 1993)

\begin{abstract}
Results on the systematics of the magnetic penetration depth $\lambda_{a b}$ in the high-temperature superconductors $\mathrm{Bi}_{2} \mathrm{Sr}_{2} \mathrm{Ca}_{1-z} \mathrm{Y}_{z} \mathrm{Cu}_{2} \mathrm{O}_{8+\delta} \quad(z=0,0.1,0.2,0.3,0.4,0.45)$ and $\mathrm{Bi}_{2-x} \mathrm{~Pb}_{x} \mathrm{Sr}_{2} \mathrm{CaCu}_{2} \mathrm{O}_{8+\delta}$ $(x=0.15,0.30,0.70)$ are reported from muon-spin-rotation measurements on polycrystalline samples with known oxygen excess $\delta$. In determining $\lambda_{a b}$ various additional sources for inhomogeneous internal field distributions, besides the one arising from the flux-line lattice, have been critically taken into account. The most important one arises from a type of powder broadening due to the anisotropy of the field-cooled magnetization. It is found that $\lambda_{a b}$ in the Y-doped compounds correlates in the expected manner with the nominal hole-carrier concentration $p\left(\lambda_{a b}^{-2} \propto p\right)$ while the Pb-doped compounds $\lambda_{a b}$ is practically independent of $p$. It is concluded that $\mathrm{Pb}$ doping (valence state is +2 ) does not increase the density of free charge carriers in agreement with results from Hall-effect measurements. No correlation between $\lambda_{a b}^{-2}$ and $T_{c}$ in the manner seen in the 1:2:3 family is found in the present case.
\end{abstract}

\section{INTRODUCTION}

The London or magnetic penetration depth $\lambda$ in superconductors is one of the most basic parameters as its temperature dependence can give information on the symmetry properties of the energy gap and coupling mechanism of the superconducting state and as its low-temperature value is related to the effective-mass tensor $\left(m_{i j}\right)$ and density of the superconducting carriers $n_{s}$ as follows: $:^{1,2}$

$$
\frac{1}{\lambda_{i i}^{2}}=\frac{4 \pi n_{s} e^{2}}{m_{i i} c^{2}}=\frac{4 \pi r_{e} n_{s}}{m_{i i} / m_{0}},
$$

where the index $i$ refers to the principal axes of the effective-mass tensor of an (anisotropic) superconductor. ( $r_{e}=$ classical electron radius, $m_{0}=$ free-electron mass). Most of the information on the magnetic penetration depth in high-temperature superconductors stems from muon-spin-rotation ${ }^{3}$ ( $\left.\mu \mathrm{SR}\right)$ measurements, which can be performed relatively easily using polycrystalline or monocrystalline material. Systematic measurements as function of composition and doping have generally been per- formed on polycrystalline material, since monocrystalline samples were usually not available. Based mainly on results from the system $\mathrm{YBa}_{2} \mathrm{Cu}_{3} \mathrm{O}_{7-\delta}$, Uemura and coworkers have postulated the existence of a universal linear relationship between $1 / \lambda^{2}$, extracted from $\mu$ SR data, and the transition temperature $T_{c}$, which seems to hold for compounds with $T_{c}<T_{c, \max } \simeq 90 \mathrm{~K}$. ${ }^{4,5}$ Interestingly, this behavior extrapolates to the system $(\mathrm{La}, \mathrm{Sr}) \mathrm{CuO}_{4}$, members of the $\mathrm{Bi}$ - or Tl-based 2:2:1:2 and $2: 2: 2: 3$ families, the bismuthates and even chevrel-phase superconductors, implying that for low carrier density compounds

$$
T_{c} \propto n_{s} / m^{*},
$$

where $m^{*}$ is the effective-carrier mass. Uemura et al. ${ }^{5}$ have suggested that these and other exotic superconductors, like certain heavy-fermion systems provide evidence for a state which interpolates between BCS pairing and Bose-Einstein condensation. On the theoretical side Schneider has shown that $T_{c} \propto 1 / \lambda^{2} \propto n_{s}$ is obtained from an approach which treats the superconductivity in high- 
$T_{c}$ systems, bismuthates, and fullerenes in close analogy to superfluid helium. ${ }^{6}$ Equation 2 seems also to be implied from the non-Ohmic $I-V$ characteristics observed in several high- $T_{c}$ families. $^{7}$

In view of the far reaching implications of the postulated universal behavior, it is important to test how universal this phenomenon really is and to locate its limitations and violations. On the experimental side, it is necessary to critically assess to what extent the $\mu$ SR data (the way they are usually analyzed) allow one to determine directly the quantity $1 / \lambda^{2}$ (see Sec. II) and what other effects could be present which could interfere with the absolute determination of $1 / \lambda^{2}$ (see Sec. III). Concerning the possible physics behind the universal scaling, it would be, moreover, extremely interesting to find out about the role of hole doping on one side and of hole filling (electron doping) on the other side. In other words one can ask if it makes a difference in which way a certain net hole concentration is established. As far as the system $\mathrm{YBa}_{2} \mathrm{Cu}_{3} \mathrm{O}_{7-\delta}$ is concerned it does not seem to make a difference, since hydrogen charging ${ }^{8}$ or partial replacement of $\mathrm{Y}$ by $\operatorname{Pr}^{9}$ (where $\mathrm{Pr}$ is in the +4 ionic state) or an increasing oxygen deficiency produce results which are all compatible with the universal curve. No such systematic tests have yet been undertaken in the Bi-based high- $T_{c}$ families. Given this and the fact that only two different members of the 2:2:1:2 family are included in the data basis of Ref. 5, we have undertaken to investigate by $\mu$ SR the system

$$
\mathrm{Bi}_{2-x} \mathrm{~Pb}_{x} \mathrm{Sr}_{2} \mathrm{Ca}_{1-z} \mathrm{Y}_{z} \mathrm{Cu}_{2} \mathrm{O}_{8+\delta} \text {, }
$$

by changing $x$ and $z$ in a systematic way, determining in each case also the oxygen excess $\delta$. Assigning the valence state +3 to $\mathrm{Bi},+2$ to $\mathrm{Pb},+2$ to $\mathrm{Sr},+2$ to $\mathrm{Ca},+3$ to $\mathrm{Y}$, and -2 to oxygen, charge neutrality requires for $\mathrm{Cu}$ the valence state $2+p$, where $p$, the hole concentration per $\mathrm{Cu}$ atom, is given by

$$
p=\delta+\frac{x-z}{2} \text {. }
$$

$\mathrm{Pb}$ doping leads thus to additional holes, while $\mathrm{Y}$-doping diminishes the number of holes. The oxygen excess $\delta$ is the primary source for holes. Hall-effect measurements in the Y-doped compounds show that the actual hole concentration scales well with $p .^{10}$ This is not so in the $\mathrm{Pb}$-doped compounds, as we will discuss later.

It is well recognized that the Bi-based high- $T_{c}$ compounds are extremely anisotropic owing to their enhanced two-dimensional character. Measurements of the anisotropy of $H_{c 1}, H_{c 2}$, and torque measurements lead to effective-mass ratios of $400 \lesssim m_{c} / m_{a b} \lesssim 10^{5}, 11-17$ where $m_{c}$ is the effective-mass for carriers moving along the crystallographic $c$ axis and $m_{a b}$ the effective mass for carriers moving in the basal $(a, b)$ plane. This translates into anisotropies of the penetration depth of

$$
20 \leq \lambda_{c} / \lambda_{a b} \leq 320 \text {. }
$$

Considering only results from torque measurements, ${ }^{11,16,17}$ which are believed to be the most reliable ones, owing to their weak sensitivity to flux pinning, one finds $\lambda_{c} / \lambda_{a b} \gtrsim 55$. This value will be used in the data analysis, to be described in Sec. V.

The phase diagram ( $T_{c}$ versus hole concentration) of the 2:2:1:2 family has been studied extensively by Groen, de Leeuw, and Feiner. ${ }^{18}$ It is shown there that superconductivity is absent for hole concentrations $p$ below 0.06 . Above this concentration, $T_{c}$ rises in a highly nonlinear fashion and reaches a maximum around 0.15-0.2 holes/Cu. Recently the dome-shaped $T_{c}$ versus $p$ curve has been explained by Dallacasa and Feduzi ${ }^{19}$ in terms of a quantum percolation model. In the low carrier concentration range they find approximately $T_{c} \propto n / m^{*}$, in qualitative agreement with the observations of Uemura et al. ${ }^{5}$ and others. ${ }^{20}$

Our results on the 2:2:1:2 family, to be presented and discussed below, do not follow the universal curve. Although the carrier or hole concentration changes by a factor of almost 2 (judging from both the behavior of $1 / \lambda_{a b}^{2}$ and the formal Cu valency) $T_{c}$ stays nearly constant. Only at much smaller carrier concentrations is a decrease of $T_{c}$ also observed ${ }^{44}$ (see Fig. 19).

This paper is organized as follows: In Sec. II we discuss the $\mu \mathrm{SR}$-frequency spectrum arising from the fluxline lattice of an anisotropic superconductor, in Sec. III we consider other contributions to the $\mu$ SR-frequency spectrum, in particular the role of the anisotropic magnetization leading to "powder broadening" in a polycrystalline specimen. In Sec. IV we describe the measurements and present the results in a particular rationalized form, in Sec. $\mathrm{V}$ we enter into a detailed analysis of the results, which in the end allows one to determine the London penetration depth. The dependence of the penetration depth on the particular composition of the samples is discussed in Sec. VI. The paper ends with a summary.

This work supersedes previous preliminary reports [Hyperfine Interactions 63, 93 (1990); Supercond. Sci. Technol. 4, S403 (1991), Physica C 185-189, 749 (1991) 185-189, 1093 (1991)].

\section{MAGNETIC-FLUX DISTRIBUTION IN THE MIXED OR ABRIKOSOV STATE OF AN ANISOTROPIC SUPERCONDUCTOR AND THE $\mu^{+}$FREQUENCY SPECTRUM}

The magnetic-flux distribution $\mathbf{h}(\mathbf{r})$ arising from the flux-line or vortex lattice in the mixed state of a type-II superconductor can be well treated within the London theory provided that the Ginzburg-Landau parameter $\kappa$ is large and that the external field is small in comparison to $H_{c 2}$. Both conditions are naturally fulfilled in the high- $T_{c}$ cuprate superconductors. Following Thiemann, Radovic, and Kogan ${ }^{2}$ the anisotropy in the penetration depth $\lambda$ is taken into account by introducing a phenomenological dimensionless tensor

$$
\left(\widetilde{m}_{i k}\right)=\left(\begin{array}{ccc}
\widetilde{m}_{1} \cos ^{2} \theta+\widetilde{m}_{3} \sin ^{2} \theta & 0 & \left(\widetilde{m}_{1}-\widetilde{m}_{3}\right) \sin \theta \cos \theta \\
0 & m_{1} & 0 \\
\left(\widetilde{m}_{1}-\widetilde{m}_{3}\right) \sin \theta \cos \theta & 0 & \widetilde{m}_{1} \sin ^{2} \theta+\widetilde{m}_{3} \cos ^{2} \theta
\end{array}\right)
$$


into the London theory, where the $\widetilde{m}_{i}$ are normalized elements derived from the corresponding effective-mass tensor [i.e., $\quad \widetilde{m}_{i}=m_{i} / \bar{m}, \bar{m}=\left(m_{1}^{2}, m_{3}\right)^{1 / 3}$ is the average effective mass, hence $\left.\left(\widetilde{m}_{1}^{2} \cdot \widetilde{m}_{3}\right)^{1 / 3}=1\right]$. $\theta$ denotes the angle between the average magnetic induction $B=\langle\mathbf{h}(r)\rangle$ (defining the $z$ axis) and the crystallographic $c$ axis of uniaxial symmetry. $\widetilde{m}_{1}=\widetilde{m}_{X X}=\widetilde{m}_{Y Y}$ and $\widetilde{m}_{3}=\widetilde{m}_{Z Z}$ are the principal values of the tensor in a coordinate system $(X Y Z)$ fixed to the main crystal axes $(Z \| c$ axis). The penetration depth along the $c$ axis and in the basal $(a, b)$ plane are then given by

$$
\begin{aligned}
& \lambda_{c}=\sqrt{\tilde{m}_{3}} \lambda, \\
& \lambda_{a b}=\sqrt{\tilde{m}_{1}} \lambda,
\end{aligned}
$$

with

$$
\lambda=\sqrt{\bar{m} c^{2} / 4 \pi e^{2} n_{s}} .
$$

The periodic flux distribution $\mathbf{h}(\mathbf{r})$ can most generally be expressed as a Fourier sum

$$
\mathbf{h}(\mathbf{r})=\sum_{\mathbf{G}} \mathbf{h}(\mathbf{G}) \exp (i \mathbf{G} \cdot \mathbf{r}),
$$

where $\mathbf{G}$ are lattice vectors of the reciprocal flux-line lattice. The Fourier components $\mathbf{h}(\mathbf{G})$ follow the London equations and are given by

$$
\begin{aligned}
& h_{x}(\mathbf{G})=B \lambda^{2} \widetilde{m}_{x z} G_{y}^{2} / d, \\
& h_{y}(\mathbf{G})=-B \lambda^{2} \widetilde{m}_{x z} G_{x} G_{y} / d, \\
& h_{z}(\mathbf{G})=B\left(1+\lambda^{2} \widetilde{m}_{z z} G^{2}\right) / d,
\end{aligned}
$$

with

$$
\begin{aligned}
d= & \left(1+\lambda^{2} \widetilde{m}_{1} G_{x}^{2}+\lambda^{2} \widetilde{m}_{x x} G_{y}^{2}\right)\left(1+\lambda^{2} \widetilde{m}_{z z} G^{2}\right) \\
& -\lambda^{4} \widetilde{m}_{x z}^{2} G^{2} G_{y}^{2}
\end{aligned}
$$

and

$$
\begin{aligned}
& G_{x}=\pi\left(\frac{2 B}{\phi_{0}}\right)^{1 / 2}\left(\frac{3 \tilde{m}_{3}}{\widetilde{m}_{z z}}\right)^{1 / 4} n, \\
& G_{y}=\pi\left(\frac{2 B}{\phi_{0}}\right)^{1 / 2}\left[\frac{\widetilde{m}_{z z}}{3 \tilde{m}_{3}}\right]^{1 / 4}(2 m-n), \\
& m, n=0 \pm 1, \pm 2, \ldots .
\end{aligned}
$$

Figure 1 displays contour plots of $h(\mathbf{r})$ calculated with the help of Eqs. (7)-(10) for $B=3000 \mathrm{G}, \lambda_{c} / \lambda_{a b}=7$, $\lambda_{a b}=1700 \AA, \xi=15 \AA$. [In order to speed up the convergence of the sum Eq. (7), each term in Eq. (4) has been multiplied by $\exp \left\{-\xi^{2}\left(G_{x}^{2} / \widetilde{m}_{x x}+G_{y}^{2} / \widetilde{m}_{y y}\right) / 2\right\}$. This procedure results also in a better description of the magnetic induction in the center of a fluxoid, which is not so well accounted for in the London theory. ${ }^{21}$ ] Equations (8) teach that for $\mathbf{B}$ not parallel to one of the principal axes of the mass tensor, nonzero transverse field components $h_{x}$ and $h_{y}$ will be present, which will tilt the local fields $\mathbf{h}(\mathbf{r})$ away from the induction $\mathbf{B}$. This deviation of $\mathbf{h}(\mathbf{r})$ from being parallel to $\mathbf{B}$ in the general case has to be taken into account if one now considers the precessional

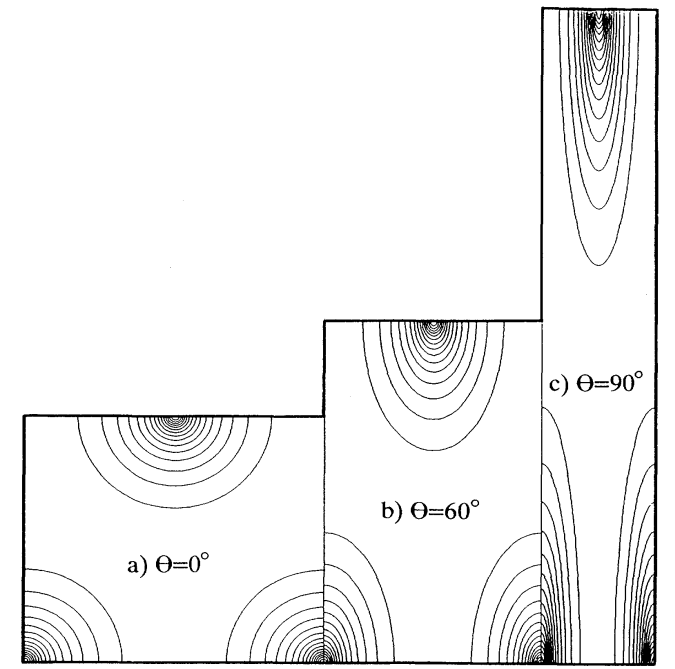

FIG. 1. Calculated contour plot of the local magnetic induction $h(\mathbf{r})$ in an anisotropic superconductor for various directions of the average induction $B$ with respect to the principal axis of an axially symmetric mass tensor $\left(=c\right.$ axis in the high- $T_{c}$ superconductors). The lines were calculated for $B=3 \mathrm{kG}$, $\lambda_{c} / \lambda_{a b}=7, \lambda_{a b}=1700 \AA$, and $\xi=15 \AA$.

motion of the implanted $\mu^{+}$spin in the field $\mathbf{h}(\mathbf{r})$. To this end we consider the following arrangement: The initial $\mu^{+}$polarization $\mathbf{P}_{\mu}(0)$ (defining the $x$ direction, $\left.\left|\mathbf{P}_{\mu}(0)\right|=1\right)$ is perpendicular to the external field, i.e., effectively perpendicular to $\mathbf{B}$. As long as $\mathbf{h}(\mathbf{r})$ is also perpendicular to $\mathbf{P}_{\mu}(0)$ the $\mu^{+}$spins will precess in a plane perpendicular to $\mathbf{h}(\mathbf{r})$. However, if $\mathbf{h}(\mathbf{r})$ and $\mathbf{P}_{\mu}(0)$ enclose an angle different from $90^{\circ}$, the $\mu^{+}$spins will precess on the surface of a cone. If the evolution of the $\mu^{+}$ polarization is observed along the $x$ direction, (i.e., the decay positrons are monitored along that direction) $P_{x}(t)$ is given by

$$
\begin{aligned}
& P_{x}(\mathbf{r}, t)=\frac{1}{\left[h(\mathbf{r}) \cdot P_{\mu}(0)\right]^{2}}\left\{\left[\mathbf{h}(r) \cdot \mathbf{P}_{\mu}(0)\right]^{2}\right. \\
&\left.+\left[\mathbf{h}(r) \times \mathbf{P}_{\mu}(0)\right]^{2} \cos \omega t\right\} \\
&=h_{x}^{2} / h^{2}+\left(1-h_{x}^{2} / h^{2}\right) \cos \omega_{\mu} t,
\end{aligned}
$$

where

$$
\omega_{\mu}=\gamma_{\mu} h(\mathbf{r}) .
$$

Consequently, in calculating the spectral distribution $F\left(\omega_{\mu}\right)$ a particular $\omega_{\mu}(\mathbf{r})$ has to be weighted by the factor $\left[1-h_{x}^{2}(\mathbf{r}) / h^{2}(\mathbf{r})\right]$. The spectral distribution can then be expressed by the formula

$$
F(\omega)=\frac{1}{S} \iint \gamma_{\mu}\left[1-\left[\frac{h_{x}(\mathbf{r})}{h(\mathbf{r})}\right]^{2}\right] \delta(h-h(\mathbf{r})) d^{2} r,
$$

where $\delta(h-h(\mathbf{r}))$ denotes the Dirac $\delta$ function and the integral extends over the area $S$ of the two-dimensional unit cell of the flux-line lattice. Figure 2 displays $F_{\theta}(\omega)$ calculated with the same parameters as the contour plot 


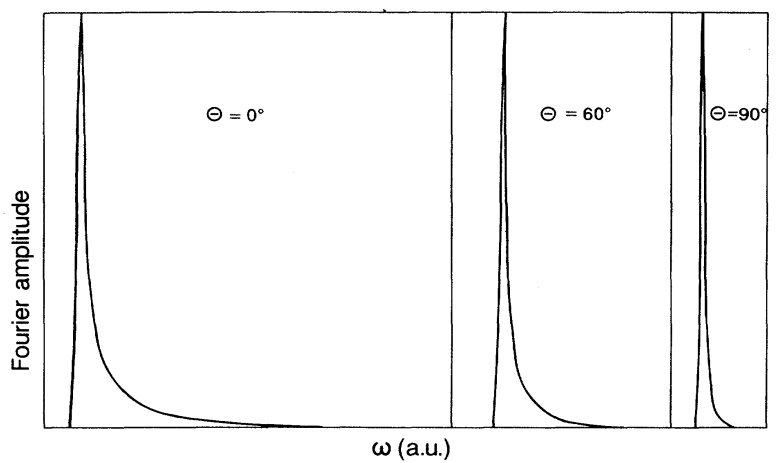

FIG. 2. Spectral distributions $F(\omega)$ of $\mu^{+}$precession frequencies in monocrystalline material following from the spatial distributions shown in Fig. 1. The indicated angles refer again to the directions of the average magnetic induction $B$ with respect to the $c$ axis. As can be seen the overall width of $F(\omega)$ depends very much on the orientation and reflects the anisotropy of the mass tensor.

in Fig. 1. The spectra in Fig. 2 reflect the true ratios of the width of $F(\omega)$ for different $\theta$. As is well known the spectra are characterized by three Van Hove singularities appearing at the saddle-point field (logarithmic singularity) and at the minimum and maximum fields (steplike singularities).

In most cases $\mu \mathrm{SR}$ measurements have been performed in polycrystalline material. The above considerations apply to monocrystalline material. The spectral distribution in polycrystalline material, $\left\langle F_{\theta}(\omega)\right\rangle_{\text {poly }}$, can be calculated by performing a proper average over all possible orientations of the crystallites. As an example, Fig. 3(a) shows $\left\langle F_{\theta}(\omega)\right\rangle_{\text {poly }}$ calculated for $\lambda_{a b}=2000 \AA$ and $\lambda_{c} / \lambda_{a b}=55$. A characteristic feature of a polycrystalline spectral distribution is the shoulder on the low-frequency side of $\left\langle F_{\theta}(\omega)\right\rangle_{\text {poly }}$.

The second moment $\left\langle\Delta \omega^{2}\right\rangle$ of the frequency distribution $F_{\theta}(\omega)$ is easily calculated. ${ }^{21,22}$ For $\theta=0^{\circ}$ and $\theta=90^{\circ}$ one finds ${ }^{23}$

$$
\begin{aligned}
\left\langle\Delta \omega^{2}\right\rangle_{\theta=0} & \left.=\gamma_{\mu}^{2}<\Delta h(\mathbf{r})^{2}\right\rangle_{\theta=0} \\
& =0.0037 \gamma_{\mu}^{2} \phi_{0}^{2} / \lambda_{a b}^{4}, \\
\left\langle\Delta \omega^{2}\right\rangle_{\theta=90^{\circ}} & =0.0037 \gamma_{\mu}^{2} \phi_{0}^{2} / \lambda_{a b}^{2} \lambda_{c}^{2} .
\end{aligned}
$$

For other orientations one has again to take into account that $\mathbf{P}_{\mu}(0)$ and $\mathbf{h}(\mathbf{r})$ are not necessarily perpendicular to each other. In this case one finds ${ }^{23}$

$$
\left\langle\Delta \omega^{2}\right\rangle_{\theta}=\gamma_{\mu}^{2}\left\{\langle\Delta h(\mathbf{r})\rangle+B^{2}\right\}-\langle\omega\rangle_{\theta}^{2},
$$

where

$$
\langle\omega\rangle_{\theta}^{2}=\gamma_{\mu}^{2}\left\langle h(\mathbf{r})\left(1-\left[\frac{h_{x}(\mathbf{r})}{h(\mathbf{r})}\right)^{2}\right)\right\rangle^{2},
$$

which, according to Fesenko, Gorbunov, and Smilga ${ }^{24}$ can be expressed more conveniently by

$$
\left\langle\Delta \omega^{2}\right\rangle_{\theta}=\frac{\widetilde{m}_{z z}}{\widetilde{m}_{3}}\left\langle\Delta \omega^{2}\right\rangle_{\theta=0} .
$$

The latter expression can be used to calculate the polycrystalline average of $\left\langle\Delta \omega^{2}\right\rangle_{\theta}$ :

$$
\left\langle\Delta \omega^{2}\right\rangle_{\text {poly }}=\frac{0.0037 \phi_{0}^{2} \gamma_{\mu}^{2}}{\lambda_{a b}^{4}} \frac{\widetilde{m}_{3}+2 \widetilde{m}_{1}}{3 \widetilde{m}_{3}} .
$$

For very strong anisotropy $\left(m_{3} / m_{1} \rightarrow \infty\right)$ one arrives at the approximation ${ }^{24}$

$$
\left\langle\Delta \omega^{2}\right\rangle_{\text {poly }} \simeq \frac{0.0037 \phi_{0}^{2} \gamma_{\mu}^{2}}{3 \lambda_{a b}^{4}} .
$$

The time evolution of the $\mu^{+}$polarization, $P_{\mu}(t)$, of an ensemble of $\mu^{+}$exposed to $\mathbf{h}(\mathbf{r})$ [as before, the initial polarization $\mathbf{P}_{\mu}(0)$ is assumed to be perpendicular to $\mathbf{B}=\langle\mathbf{h}(\mathbf{r})\rangle$ and $\boldsymbol{P}_{\mu}(t)$ is observed in the direction of $\left.\mathbf{P}_{\mu}(0)\right]$ is given by the Fourier transform of $F_{\theta}(\omega)$, i.e.,

$$
P_{\mu}(\theta, t)=\operatorname{Re}\left\{\frac{1}{2 \pi} \int_{0}^{\infty} F_{\theta}(\omega) e^{i \omega t} d \omega\right\}
$$

Given the complicated form of $F_{\theta}(\omega), P_{\theta}(t)$ does not follow a simple law, such as an exponential or Gaussian decay function. Nevertheless in a majority of cases experimental data have been analyzed by assuming $P(t)$ to be of a Gaussian form [i.e., $P(t)=\exp \left(-\frac{1}{2} \sigma^{2} t^{2}\right)$ ] identifying the decay constant $\sigma^{2}$ with the second moment expressed

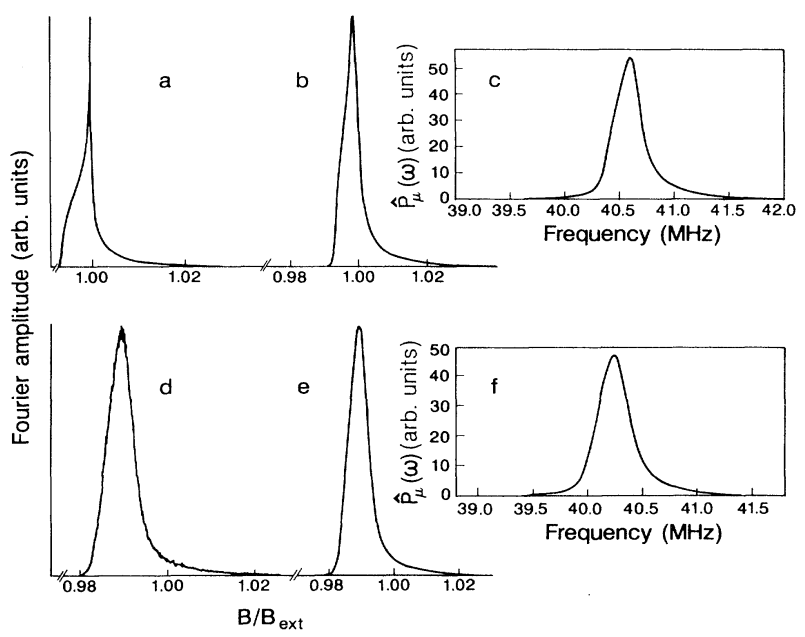

FIG. 3. Calculated spectral distributions of local magnetic fields in a polycrystalline anisotropic superconductor with $\lambda_{a b}=2000 \AA, \lambda_{c} / \lambda_{a b}=55$ : (a) distribution arising only from the vortex lattice; (b) vortex lattice plus nuclear dipolar fields $\left(\left\langle\Delta B_{\text {dip }}^{2}\right\rangle^{1 / 2}=1.88 \mathrm{G}\right)$; (c) actual frequency distribution, $\widehat{P}(\omega)$ following from a Fourier transform of a generated $\mu$ SR signal based on the distribution (b). The difference in shape between (b) and (c) arises from the finite time window ( $\sim 8 \mu \mathrm{s})$ of a realistic $\mu$ SR signal and consequently limited integration range in calculating the Fourier spectrum; (d) vortex lattice plus distribution in demagnetization factors assuming $\Delta N_{G}=4 \pi / 8$ and an isotropic $M(\theta)=-3 \mathrm{emu} / \mathrm{cm}^{3}$; (e) like in (d) plus nuclear dipole fields; ( $f$ ) actual frequency distribution of the Fourier transformed $\mu \mathrm{SR}$ signal based on (e) like in (c). 
in Eqs. (14), (15), and (19). This can give rise to rather erroneous determinations of $\lambda_{a b}$. For instance we have generated an artificial $\mu \mathrm{SR}$ spectrum for a field distribution associated with $\lambda_{a b}=2000 \AA$ and have then fitted it with a Gaussian decay function, resulting in a $\lambda_{a b}$ of 3060 $\AA$. Fortunately the situation is not as bad in practice as suggested by this example, due to other contributions to the overall field distribution in the mixed state which tend to smear out the Van Hove singularities in $F\left(\omega_{\mu}\right)$, rendering $F(\omega)$ more symmetrical.

One important source for a smearing-out effect consists in static random small displacements of the vortices out of their rigid-lattice positions. As shown by Brandt, ${ }^{25}$ this can be approximately accounted for by convoluting the rigid-lattice spectral distribution $F(\omega)$ with a Gaussian distribution of width $\widetilde{\sigma}_{a}$. According to Ref. [25], an average displacement of $3 \%$ will lead to a width $\widetilde{\sigma}_{a} \simeq 0.2 M$, where $M$ is the $\mathrm{n}$ ignetization of the sample in the mixed state. The spectral distribution in the case of complete random order of the vortex lines (vortex glass) has, to our knowledge, not been treated in the literature yet. It can be expected that the distribution will assume a rather symmetric shape. However, Portis ${ }^{26}$ has calculated the second moment for a vortex glass, assuming that the vortex density is homogeneous over areas larger than the two-dimensional unit cell. In the limit of high vortex densities for an isotropic superconductor he obtained

$$
\left\langle\Delta \omega^{2}\right\rangle=0.0063 \gamma_{\mu}^{2} \phi^{2} / \lambda^{4},
$$

which is about $70 \%$ larger than in the case of a triangular rigid vortex lattice.

In addition to the lateral disorder of an otherwise rigidly arranged lattice of parallel running flux tubes, as just discussed, also some disorder along the $\mathbf{B}$ direction can be expected to occur in a highly anisotropic superconductor with extremely small coherence length as in the case of the Bi-based high- $T_{c}$ families, in which the distance of the superconducting $\mathrm{CuO}_{2}$ planes $(\sim 3.4$ and $12 \AA$ in the 2:2:1:2 family) is much larger than the coherence length along the $c$ axis $(\sim 2 \AA)$. The resulting arrangement of "pancake"-like vortex disks may show considerable phase shifts from plane to plane leading to field variations also along the $\mathbf{B}$ direction. However, since the average lateral distance between vortex disks $(877 \AA$ at $3 \mathrm{kG})$ is much larger than the plane distances, the misalignment of a string of disk vortices as compared with a rigid tube is probably not too severe and the resulting modification of $F(\omega)$ may again be taken into account by convoluting $F(\omega)$ with a Gaussian distribution.

Vortex motions due to thermally induced vibrations and depinning are another source of smoothing of the spectral distribution $F(\omega)$. This will be primarily of importance at higher temperatures and could be a very important contribution to the temperature dependence of the $\mu^{+}$relaxation rate in addition to the temperature dependence arising from the penetration depth. At low temperature, which we are primarily interested in, such effects can probably be neglected. ${ }^{27}$

\section{OTHER CONTRIBUTIONS \\ TO THE FLUX DISTRIBUTION IN A POLYCRYSTALLINE SAMPLE}

In this section we discuss additional sources of nonhomogeneous flux distributions with particular reference to polycrystalline samples. These sources are (1) nuclear dipole fields (also important in single crystals), (2) anisotropic magnetization in the superconducting state, (3) distribution in demagnetization factors, (4) texture, and (5) critical state.

\section{A. Nuclear dipole fields}

The presence of $\mathrm{Cu}$ nuclei $\left({ }^{63,65} \mathrm{Cu}\right)$ with nonzero magnetic dipole moments in all high- $T_{c}$ superconductors gives rise to a distribution of dipolar fields, the spectral distribution of which is Gaussian. ${ }^{28} F(\omega)$ has to be convoluted with this distribution. The effect of nuclear dipole fields is therefore similar to the effect of slight random displacements of the vortices or vortex disks from their regular periodic positions as discussed above. The nuclear dipolar field distribution width $\left\langle\Delta B_{\text {dip }}^{2}\right\rangle^{1 / 2}$ can be determined from the $\mu^{+}$-spin relaxation just above $T_{c}$. In the Bi-2:2:1:2 family members it amounts to $\left\langle\Delta B_{\text {dip }}^{2}\right\rangle^{1 / 2} \simeq 1.88 \mathrm{G}$. In all following calculations of the spectral distribution this contribution is generally folded in. Its effect is seen in Figs. 3(b), 3(e), 6(b), 6(e), and 6(h).

\section{B. Anisotropic magnetization}

It is well known that the induced magnetization in the superconducting state of a high- $T_{c}$ compound is very anisotropic. $^{29}$ This has pronounced consequences for the field distribution in a polycrystalline or granular sample. ${ }^{30}$ Let us consider the local magnetic induction of the local average field sensed by the $\mu^{+}$sitting in a particular grain:

$$
\begin{aligned}
\mathbf{B}_{\mu}(\theta)= & \mathbf{B}_{\mathrm{ext}}-\rho_{P} N_{P} \mathbf{M}_{P}+\rho_{P} N_{H} \mathbf{M}_{P}+4 \pi \rho_{G} \mathbf{M}(\theta) \\
& -\rho_{G} N_{G} \mathbf{M}(\theta) .
\end{aligned}
$$

The various terms have the following meaning: $\quad \mathbf{B}_{\text {ext }}=$ external field, $-\rho_{P} N_{P} \mathbf{M}_{P}$ is the demagnetization field of the whole polycrystalline sample $\left[N_{P}=\right.$ demagnetization factor, $\mathbf{M}_{P}=\langle\mathbf{M}(\theta)\rangle$ $=(1 / 4 \pi) \int_{\Omega} \mathbf{M}(\theta) d \Omega$ is the sample magnetization, $\rho_{P}$ is the crystalline density, $\rho_{G}$ is the effective volume density, $\mathbf{M}(\theta)$ is the crystalline Meissner magnetization, $\mathbf{M}_{P}, \mathbf{M}(\theta)$ are always measured in emu/g], $+\rho_{P} N_{H} \mathbf{M}_{P}$ is the field associated with the hole that would emerge when the $\mu^{+}$containing grain is removed, $4 \pi \rho_{G} \mathbf{M}(\theta)$ is the induction due to the Meissner magnetization of this grain, and $-\rho_{G} N_{G} \mathbf{M}_{G}(\theta)$ its demagnetization field. Performing an average over all grains it is seen that the last three terms will give rise to a spread in local fields. The most serious contribution is associated with the anisotropy of $\mathbf{M}(\theta) . \theta$ is defined as in Eq. (4).

If $M(\theta)$ in our field-cooling procedure behaves like the remanent magnetization $M_{\text {rem }}(\theta)$, one expects ${ }^{31}$

$$
M(\theta)=M_{0}+M_{1} \cos \theta \text {. }
$$


If, on the other hand, $M(\theta)$ is given by the ansatz

$$
\mathbf{M}=\overleftrightarrow{\chi} \cdot \mathbf{H}_{\mathrm{ext}},
$$

where $\chi$ is a traceless susceptibility tensor, $M(\theta)$ should follow the expression

$$
M(\theta)=M_{0}+M_{1} P_{2}^{0}(\cos \theta) .
$$

Measurements of the angular dependence of the fieldcooled magnetization in a $\mathrm{Bi}_{2.2} \mathrm{Sr}_{1.7} \mathrm{CaCu}_{2} \mathrm{O}_{8+\delta}$ single crystal by Niskanen ${ }^{32}$ (see Fig. 4) could also suggest a dependence of the form

$$
M(\theta)=M_{0}+M_{1} \theta .
$$

The spectral distribution of $M(\theta)$ in a polycrystal follows from

$$
F(M)=\sin \theta\left|\frac{d M}{d \theta}\right|^{-1}
$$

and is shown in Fig. 5 for all three cases. The distribution $F(M)$ is taken into account in the calculation of the overall polycrystalline distribution $\left\langle F_{\theta}(\omega)\right\rangle_{\text {poly }}$ by shifting the average frequency of each $F_{\theta}(\omega)$ according to Eq. (23), using for $M(\theta)$ either Eqs. (24), (26), or (27), before averaging properly over all crystal orientations. Figure 6 displays distributions calculated in this way (for details see the figure caption). For a comparison with the distribution for $M(\theta) \equiv 0$, see Fig. 3(a). As can be seen, the four spectra differ significantly from each other.

\section{Demagnetization factor}

In a polycrystalline granular sample it can be expected that the grains show a wide distribution of shapes. This together with a random orientation of the grains should result in a distribution of demagnetization factors $N_{G}$. We approximate this distribution by a Gaussian distribution,

$$
F\left(N_{G}\right)=\frac{1}{\Delta N_{G} \sqrt{2 \pi}} \exp \left\{-\frac{\left(N_{G}-4 \pi / 3\right)^{2}}{2 \Delta N_{G}^{2}}\right\} .
$$

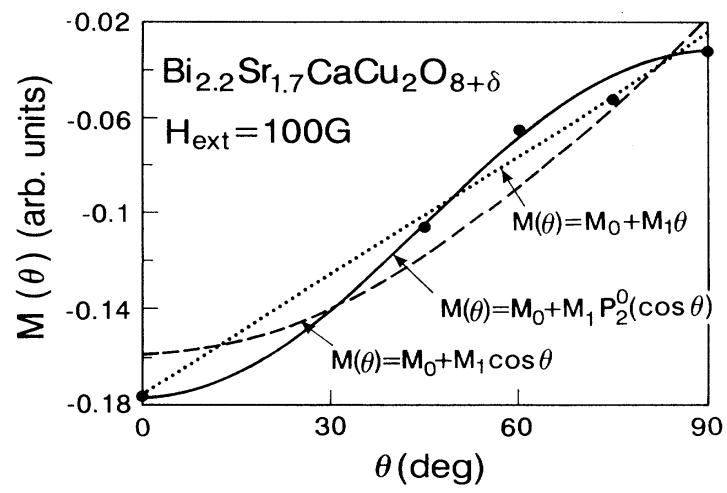

FIG. 4. Angular dependence of field-cooled magnetization in a $\mathrm{Bi}_{2.2} \mathrm{Sr}_{1.7} \mathrm{CaCu}_{2} \mathrm{O}_{8+\delta}$ single crystal (closed circles) at $20 \mathrm{~K}$ and $100 \mathrm{G}$ (courtesy of $\mathrm{K}$. Niskanen). The solid lines represent various fits to the data. $\theta$ is the angle between $B_{\text {ext }}$ and the crystal$\log$ raphic $c$ axis.

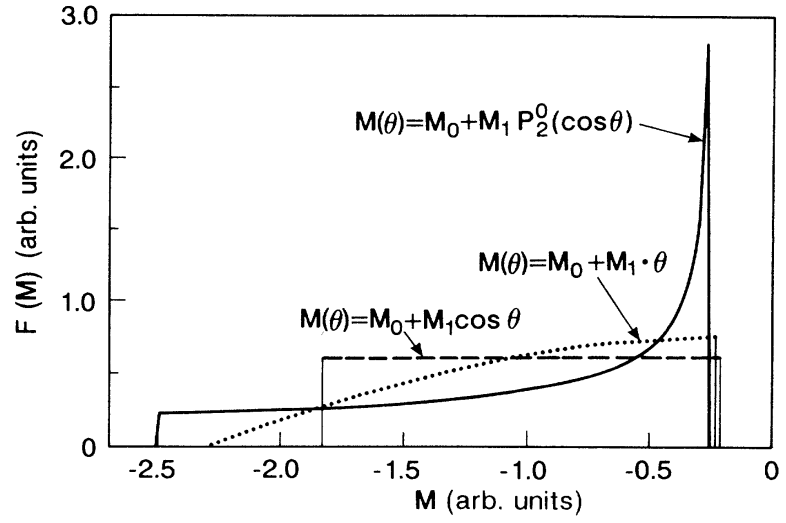

FIG. 5. Calculated spectral distributions of $\boldsymbol{M}$ associated with different angular dependencies of $M(\theta)$ [Eqs. (24), (26), and (27)].

Measurements of the powder broadening of the $\mu \mathrm{SR}$ linewidth in $R \mathrm{Ba}_{2} \mathrm{Cu}_{3} \mathrm{O}_{7}(R=\mathrm{Ho}, \mathrm{Er}, \mathrm{Dy})$ above $T_{c}$ are also affected by the distribution of $N_{G}$. We find from an analysis of such data that the above form for $F\left(N_{G}\right)$ appear to be a good approximation yielding moreover $\Delta N_{G} \simeq 4 \pi / 8 .^{33}$ In Fig. 6 the distribution $F\left(N_{G}\right)$ with $\Delta N_{G}=4 \pi / 8$ is generally folded in. Figure 3(d) shows the
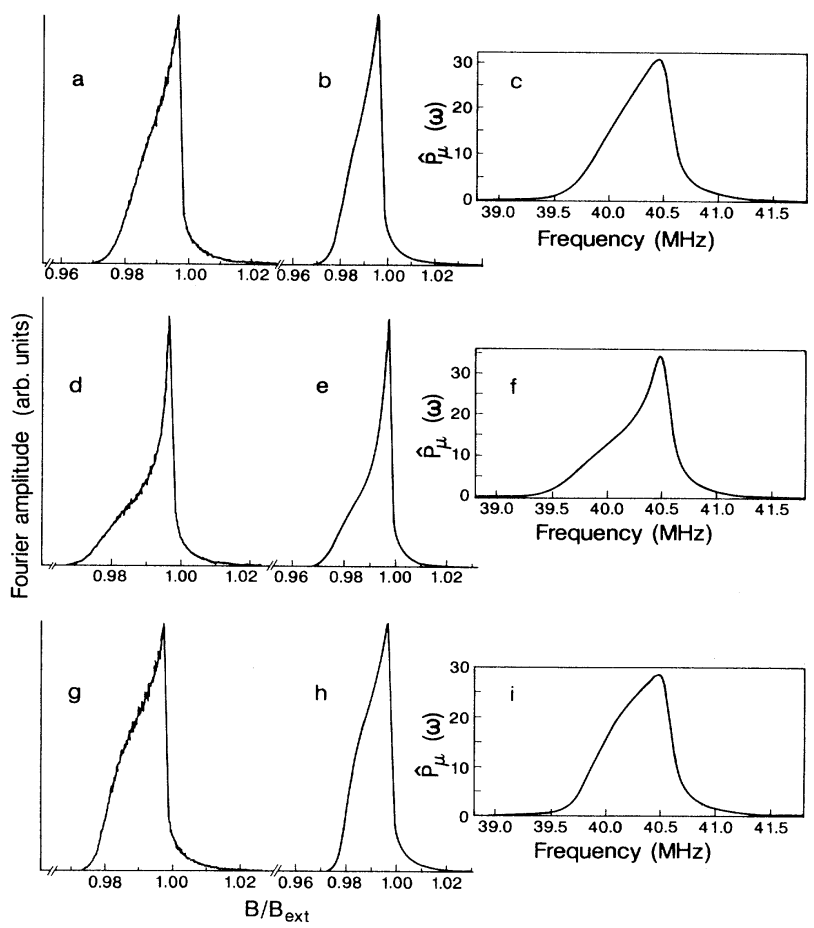

FIG. 6. Spectral distributions of local magnetic fields calculated for a polycrystalline granular anisotropic superconductor with $\lambda_{a b}=2000 \AA, \lambda_{c} / \lambda_{a b}=55, M\left(\theta=0^{\circ}\right) / M\left(\theta=90^{\circ}\right)=6.5$, and $\langle M(\theta)\rangle_{\text {poly }}=-3 \mathrm{emu} / \mathrm{cm}^{3}, \Delta N_{G}=4 \pi / 8$; (a) $M(\theta)$ is given by Eq. (24); (d) $M(\theta)=$ Eq. (26); (g) $M(\theta)=$ Eq. (27); (b), (e), and (h) include nuclear dipole field and (c), (f), and (i) are the corresponding frequency distributions $\hat{\boldsymbol{P}}(\omega)$ following from Fourier transformation of generated $\mu \mathrm{SR}$ signals [like in Figs. 3(c) and $3(\mathrm{f})]$. 
effect of such a $F\left(N_{G}\right)$ in case of an isotropic $M(\theta) \neq 0$. The result is a much more symmetric over all distribution.

\section{Texture}

Ceramic samples prepared by cold or hot pressing may contain grains with a certain preferred orientation. For example, if the grains are present in the form of thin platelets with the crystallographic $c$ axis perpendicular to the flat surface one may envision a preferred stacking of the platelets along the $c$ direction. However this effect is, if present at all, rather small. To get an idea on its influence on the $\mu^{+}$frequency distribution we assume $\theta=c \theta^{\prime}$ with $c<1$ and a distribution of $\theta^{\prime}$ given by $F\left(\theta^{\prime}\right) \propto \sin \theta^{\prime} . \theta$ is, as before, the angle between $\mathbf{B}\left(\approx \mathbf{B}_{\mathrm{ext}}\right)$ and the $c$ axis of a crystallite, i.e., $F\left(\theta^{\prime}\right)$ reflects a preferred orientation of the crystallite's $c$ axis parallel to $\mathbf{B}$. Figure 7 shows two generated spectra, one (a) without texture [same as in Fig. 6(a)], the other one (b) with texture $(c=0.8)$, as described. According to Fig. 7, modest texture, if present, leads also to a more symmetric spectral distribution.

\section{E. Critical state (Bean's model)}

Upon field cooling a nonuniform distribution of local induction $B(r)$ will evolve in a sample if pinning hinders the uniform expansion of the flux-line lattice due to diamagnetic screening currents. Assuming field-independent pinning forces the induction $B$ or the density of vortices

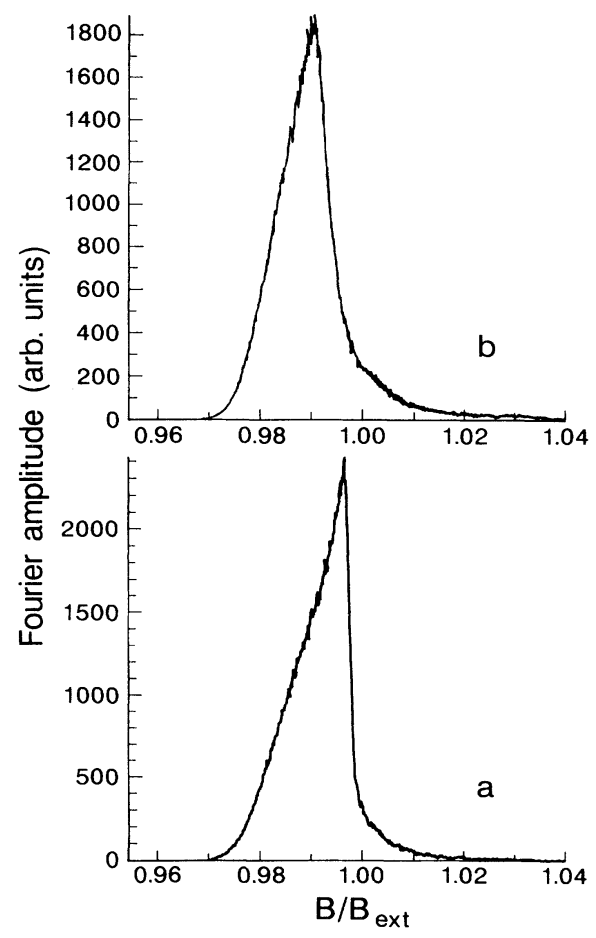

FIG. 7. Effect of texture on the spectral distribution of internal fields; (a) same as in Fig. 6(a), (b) with inclusion of texture $(c=0.8$, see text). decreases linearly with distance from the center to the edge of the grain. The resulting spectral distribution (for a cylindrical geometry) is given by $F_{C S}(B)=2\left(B_{0}-B\right) / B^{* 2}$, were $B_{0}-B^{*} \leq B \leq B_{0}$ and $B^{*}=(4 \pi / c) R \cdot J_{c}, B_{0}$ is the trapped flux at the center of the grain, $J_{c}$ is the critical current density, and $R$ is the radius of the cylinder. Variations in grain sizes and shapes in a polycrystalline sample were taken into account in Ref. 34 by averaging $F_{\mathrm{CS}}(B)$ over a Gaussian distribution in $B^{*}$. It was shown that the shape of the overall spectral distribution of $\omega$ in a ceramic $\mathrm{YBa}_{2} \mathrm{Cu}_{3} \mathrm{O}_{7}$ sample was very much influenced by $F_{\mathrm{CS}}$. Work published by the same group on measurements in $\mathrm{Bi}_{2} \mathrm{Sr}_{2} \mathrm{CaCu}_{2} \mathrm{O}_{8}$ (Ref. 35), revealed much more symmetric spectral distributions and no attempt was made to include the possibility of a nonuniform local induction distribution inside a grain into the analysis. Also the present data do not lend evidence for the importance of gradients in the flux-line density on the overall spectral distribution of $\omega$. We will, therefore, neglect this possibility in the analysis of the present data.

\section{MEASUREMENTS}

\section{A. Samples}

All samples belonging to the 2:2:1:2 family were prepared at Hoechst AG (Hürth) by a proprietary melt process, ${ }^{36}$ which starts with heating a homogeneous mixture of the binary oxides $\mathrm{Be}_{2} \mathrm{O}_{3}, \mathrm{SrO}, \mathrm{CaO}, \mathrm{CuO}$, and $\mathrm{PbO}$, respectively, $\mathrm{Y}_{2} \mathrm{O}_{3}$, to about $1050^{\circ} \mathrm{C}$ until a homogeneous melt is obtained. The melt is cast into copper molds where solidification and slow cooling to room temperature occurs. The thus obtained compact cast bodies, which are multiphased, ${ }^{36}$ are subjected to an annealing step around $850^{\circ} \mathrm{C}$ during which the superconducting two-layer phase is formed. The rapid transformation of the multiphased material is assisted by a partial melting in the bulk material during annealing. ${ }^{37}$ The oxygen content can be monitored during the annealing step by a proper adjustment of temperature and partial oxygen pressure. The oxygen content was chosen such that for each composition a maximum in $T_{c}$ was achieved. The exact metal content was subsequently determined by chemical analysis and the oxygen content by iodometric titration. The presence of foreign phases in the samples was checked by x-ray diffraction. Within the accuracy of these measurements ( $\lesssim 5 \%)$ no foreign phases could be detected.

Critical temperatures $T_{c}$ were determined by resistivity and low-field (10 G) magnetization measurements. Superconducting quantum interference device measurements served also to determine the field-cooled magnetization at $3 \mathrm{kG}$, an important parameter in the analysis of the $\mu \mathrm{SR}$ data (see Sec. V). The latter results are displayed in Figs. 8(a) and 8(b).

Table I presents a list of the investigated samples and their $T_{c}$ (onset of diamagnetic response; at this temperature the resistivity had essentially vanished) and fieldcooled $(3 \mathrm{kG})$ low-temperature magnetization $M(T \rightarrow 0)$. The oxygen excess $\delta$ is listed in Table II. 
TABLE I. List of samples, critical temperatures $T_{c}$, and low-temperature magnetization $M_{P}(\sim 10 \mathrm{~K})$ after field cooling in $3 \mathrm{kG}$.

\begin{tabular}{lcc}
\hline \hline \multicolumn{1}{c}{ Sample } & $T_{c}(\mathrm{~K})$ & $M_{P}(\sim 10 \mathrm{~K})(\mathrm{emu} / \mathrm{g})$ \\
\hline $\mathrm{Bi}_{1.30} \mathrm{~Pb}_{0.70} \mathrm{Sr}_{2} \mathrm{CaCu}_{2} \mathrm{O}_{8.28}$ & $81(1)$ & $-0.235(8)$ \\
$\mathrm{Bi}_{1.70} \mathrm{~Pb}_{0.30} \mathrm{Sr}_{2} \mathrm{CaCu}_{2} \mathrm{O}_{8.25}$ & $81(1)$ & $-0.371(8)$ \\
$\mathrm{Bi}_{1.85} \mathrm{~Pb}_{0.15} \mathrm{Sr}_{2} \mathrm{CaCu}_{2} \mathrm{O}_{8.10}$ & $91(1)$ & $-0.567(2)$ \\
& & \\
$\mathrm{Bi}_{2} \mathrm{Sr}_{2} \mathrm{CaCu}_{2} \mathrm{O}_{8.16}$ & $92(1)$ & $-0.537(2)$ \\
& & \\
$\mathrm{Bi}_{2} \mathrm{Sr}_{2} \mathrm{Ca}_{0.90} \mathrm{Y}_{0.10} \mathrm{Cu}_{2} \mathrm{O}_{8.17}$ & $89(1)$ & $-0.405(8)$ \\
$\mathrm{Bi}_{2} \mathrm{Sr}_{2} \mathrm{Ca}_{0.80} \mathrm{Y}_{0.20} \mathrm{Cu}_{2} \mathrm{O}_{8.19}$ & $88(1)$ & $-0.233(8)$ \\
$\mathrm{Bi}_{2} \mathrm{Sr}_{2} \mathrm{Ca}_{0.70} \mathrm{Y}_{0.30} \mathrm{Cu}_{2} \mathrm{O}_{8.28}$ & $86(1)$ & $-0.268(8)$ \\
$\mathrm{Bi}_{2} \mathrm{Sr}_{2} \mathrm{Ca}_{0.60} \mathrm{Y}_{0.40} \mathrm{Cu}_{2} \mathrm{O}_{8.31}$ & $85(1)$ & $-0.198(8)$ \\
$\mathrm{Bi}_{2} \mathrm{Sr}_{2} \mathrm{Ca}_{0.55} \mathrm{Y}_{0.45} \mathrm{Cu}_{2} \mathrm{O}_{8.33}$ & $84(1)$ & $-0.161(8)$ \\
\hline \hline
\end{tabular}

For the $\mu \mathrm{SR}$ measurements $50 \mathrm{~g}$ of the powderous material were cold pressed into the form of a disk with 40 $\mathrm{mm}$ diameter and 6-10 $\mathrm{mm}$ thickness. Typical densities of $\rho_{G}=4.66 \mathrm{~g} / \mathrm{cm}^{3}$ were obtained. The demagnetization factor $N_{P}$ of these disks for fields in the disk plane was determined from the ratio thickness over diameter with use of a table taken from Ref. 38 to amount to $N_{P}=(0.76 \pm 0.10) 4 \pi$.

\section{B. Data acquisition}

The $\mu$ SR measurements were performed on the muon beamline of the Phasotron of the Joint Institute for $\mathrm{Nu}$ clear Research in Dubna (Russia) using the spectrometer "MUSPIN." 39 An external field $H_{\text {ext }}$ of generally $3 \mathrm{kG}$, controlled by NMR, was applied perpendicular to the initial $\mu^{+}$polarization, i.e., perpendicular to the beamline, allowing to observe a transverse field $\mu$ SR signal ( $\mu^{+}$Larmor precession). The sample was mounted in a He-flow cryostat, which allowed us to use temperatures between $4.2 \mathrm{~K}$ and room temperature. The temperature was stabilized to better than $0.05 \mathrm{~K}$.

The $\mu$ SR signal was observed in the usual time differential way by monitoring the positron rate from the $\mu^{+}$decay as a function of elapsed $\mu^{+}$lifetime in two positron detector telescopes mounted in forward and backward direction with respect to the initial $\mu^{+}$polarization. The time dependence of the positron rate is basically given by the expression ${ }^{3}$

$$
\frac{d N_{e^{+}}}{d t}=N_{0} \frac{1}{\tau_{\mu}} e^{-t / \tau_{\mu}}[1+A G(t) \cos (\omega t+\varphi)]+B G,
$$
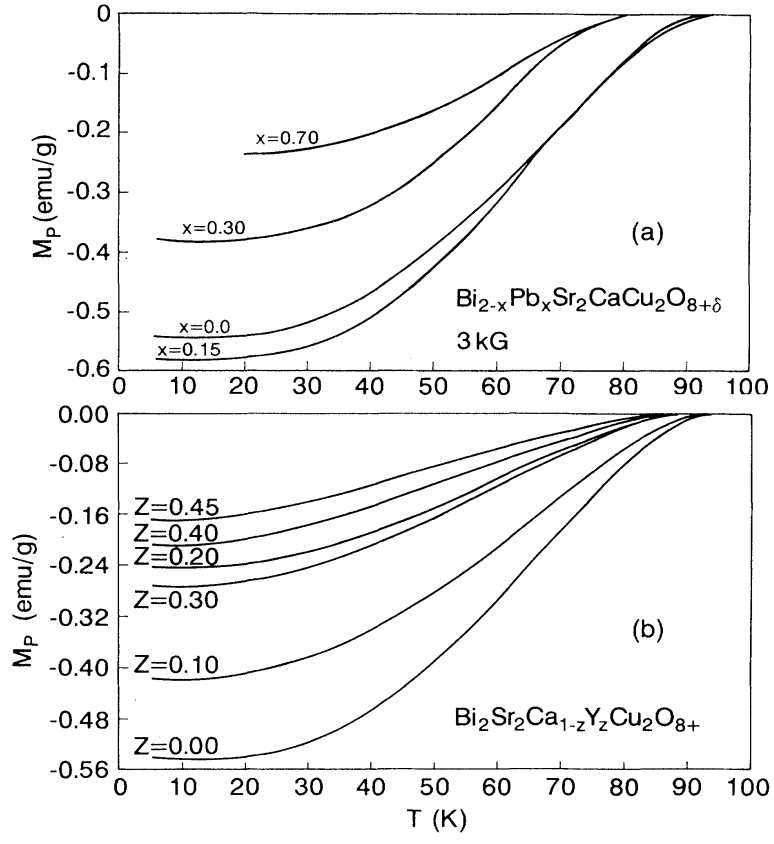

FIG. 8. Measured temperature dependence of field-cooled magnetization $\left(B_{\text {ext }}=3 \mathrm{kG}\right.$ ) for (a) the $\mathrm{Bi}_{2-x} \mathrm{~Pb}_{x} \mathrm{Sr}_{2} \mathrm{CaCu}_{2} \mathrm{O}_{8+\delta}$ compounds and (b) the $\mathrm{Bi}_{2} \mathrm{Sr}_{2} \mathrm{Ca}_{1-z} \mathrm{Y}_{z} \mathrm{Cu}_{2} \mathrm{O}_{8+\delta}$ compounds.

where $\omega=\gamma_{\mu}$. $H_{\text {ext }}$ is the Larmor frequency, $\tau_{\mu}$ is the $\mu^{+}$ lifetime, $A$ is the effective decay asymmetry ( $\simeq 10 \%$ in the present set up), and $G(t)$ is a relaxation function, the shape of which depending on the mechanisms leading to depolarization [e.g., it may follow from Eq. (21)]. $B G$ is a time-independent background which accounts for accidental decay events with $B G / N_{0} \lesssim 2 \times 10^{-3}$. As we will see later $P(t)=A G(t) \cos (\omega t+\varphi)$ may have to be replaced by a multicomponent expression $\sum_{i} A_{i} G_{i}(t) \cos \left(\omega_{i} t+\varphi\right)$ in order to describe the data more accurately. For example, a two-component expression is needed if part of the muons stop outside the sample (e.g., in the cryostat walls) where a different $\omega$ and/or a different $G(t)$ is observed.

\section{Results}

Figure 9(b) shows a typical Fourier transform (real amplitude) of the $\mu$ SR signal in time space obtained at low temperatures. It consists of a relatively narrow peak su-

TABLE II. List of "best" $\lambda_{a b}(T \simeq 10 \mathrm{~K})$ for the compounds $\mathrm{Bi}_{2-x} \mathrm{~Pb}_{x} \mathrm{Sr}_{2} \mathrm{Ca}_{1-z} \mathrm{Y}_{z} \mathrm{Cu}_{2} \mathrm{O}_{8+\delta}$.

\begin{tabular}{cccccc}
\hline \hline$x$ & $z$ & $\delta$ & $p$ & $\lambda_{a b}(\AA)$ & $n_{0}\left(m_{a b} / m_{0}\right)^{-1}\left(\mathrm{~cm}^{-3}\right)$ \\
\hline & 0.20 & 0.19 & $0.09(1)$ & $2250(150)$ & $5.6(4) \times 10^{20}$ \\
& 0.45 & 0.33 & $0.10(1)$ & $2300(150)$ & $5.3(4) \times 10^{20}$ \\
& 0.40 & 0.31 & $0.11(1)$ & $2250(150)$ & $5.6(4) \times 10^{20}$ \\
& 0.10 & 0.17 & $0.12(1)$ & $2000(150)$ & $7.0(5) \times 10^{20}$ \\
0.00 & 0.30 & 0.28 & $0.13(1)$ & $1950(150)$ & $7.4(6) \times 10^{20}$ \\
0.15 & 0.00 & 0.16 & $0.16(1)$ & $1850(100)$ & $8.3(5) \times 10^{20}$ \\
0.30 & & 0.10 & $0.18(1)$ & $1900(100)$ & $7.8(5) \times 10^{20}$ \\
0.70 & 0.25 & $0.40(1)$ & $1850(100)$ & $8.3(5) \times 10^{20}$ \\
\hline \hline
\end{tabular}




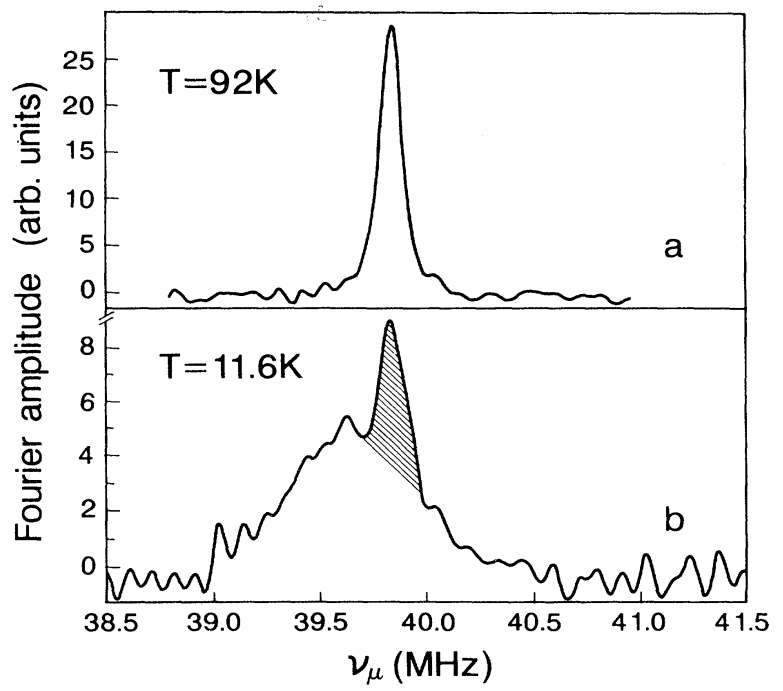

FIG. 9. Fourier spectra of the $\mu \mathrm{SR}$ signal above (a) and below (b) $T_{c}$ in $\mathrm{Bi}_{1.85} \mathrm{~Pb}_{0.15} \mathrm{Sr}_{2} \mathrm{CaCu}_{2} \mathrm{O}_{8.10}\left(B_{\text {ext }}=3 \mathrm{kG}\right)$. The broadening below $T_{c}$ is readily visible. The narrow signal superimposed on the broad signal arises from $\mu^{+}$stopped outside the sample.

perimposed on a much wider, somewhat asymmetric signal. The narrow peak arises from those muons stopping outside the sample, e.g., in the cryostat walls, etc. Its contribution to the total signal is of the order of $\sim 25 \%$. Above $T_{c}$ the Fourier transform signal is rather symmetric and the background signal is no longer visible as a distinct peak as can be seen from Fig. 9(a). For a consistent and rationalized display of the results all $\mu \mathrm{SR}$ spectra taken below $T_{c}$ were analyzed by fitting a threecomponent expression to the data (one accounting for the background signal, two for mimicking the asymmetric main signal) and all spectra taken above $T_{c}$ were analyzed by fitting a two-component expression to the data (one accounting again for the background signal, and only one for the now symmetric main signal). The background signal was most easily fitted at the lowest temperatures, where it appears as a distinct component as can be seen from Fig. 9(b). Its fitted amplitude at the lowest temperature $\left(A_{b}\right)$ was subsequently fixed in the analysis of the higher-temperature spectra. Explicitly,

$$
P_{\mu}(t)=A_{b} e^{-(1 / 2) \sigma_{b}^{2} t^{2}} \cos \left(\omega_{b} t+\phi\right)+P_{\mu}^{\mathrm{sample}}(t)
$$

with

$P_{\mu}^{\text {sample }}(t)=\sum_{j=1}^{2} A_{j} e^{-(1 / 2) \sigma_{j}^{2} t^{2}} \cos \left(\omega_{j} t+\phi\right)$ for $T<T_{c}$,

$P_{\mu}^{\text {sample }}(t)=A e^{-(1 / 2) \sigma^{2} t^{2}} \cos (\omega t+\phi)$ for $T>T_{c}$.

The same procedure was applied to the amplitude $A_{1}$ of the sample signal. It was fitted at the lowest temperature and then fixed at this value in the further analysis. As an example, Fig. 10 shows the thus obtained results for the compound $\mathrm{Bi}_{1.7} \mathrm{~Pb}_{0.3} \mathrm{Sr}_{2} \mathrm{CaCu}_{2} \mathrm{O}_{8.25}$ as a function of temperature below $T_{c}$. For comparison with results from

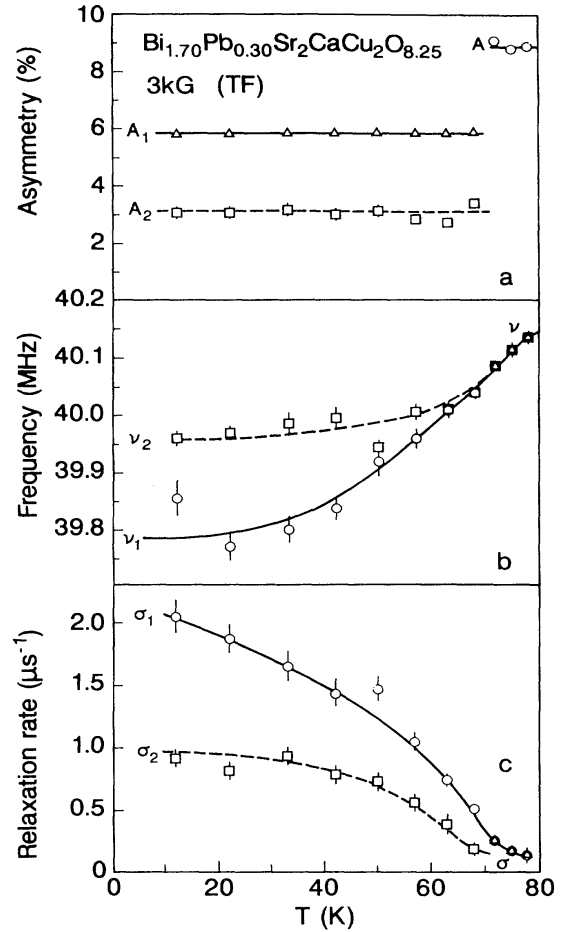

FIG. 10. Temperature dependence of the three component fit parameters up to $\sim T_{c}$ for $\mathrm{Bi}_{1.70} \mathrm{~Pb}_{0.30} \mathrm{Sr}_{2} \mathrm{CaCu}_{2} \mathrm{O}_{8.25}$; (a) asymmetries $A_{1}, A_{2}$; (b) precession frequencies $v_{1}, v_{2}$; (c) relaxation rates $\sigma_{1}, \sigma_{2}$ [see Eqs. (31) and (32)].

other groups the main signal was also fitted by only one component. The resulting $\sigma$ was used in producing Fig. 19.

Assuming that the two-component function for the main signal accounts well for the asymmetric frequency distribution seen in the Fourier spectra, the second moment of this distribution as well as the average field can be calculated from the expressions

$$
\begin{aligned}
& \left\langle\Delta \omega^{2}\right\rangle_{\mathrm{eff}}=\sum_{i=1}^{2} \frac{A_{i}}{A_{1}+A_{2}}\left\{\sigma_{i}^{2}+\left(\omega_{i}-\langle\omega\rangle\right)^{2}\right\}, \\
& \langle\omega\rangle=\sum_{i=1}^{2} \frac{A_{i} \omega_{i}}{A_{1}+A_{2}} .
\end{aligned}
$$

As an example Figs. 11 and 12 display the temperature dependence of $\left(\langle\omega\rangle-\omega_{b}\right) / 2 \pi$ and $\left\langle\Delta \omega^{2}\right\rangle_{\text {eff }}^{1 / 2}$, calculated in this way for the compound $\mathrm{Bi}_{1.85} \mathrm{~Pb}_{0.15} \mathrm{Sr}_{2} \mathrm{CaCu}_{2} \mathrm{O}_{8.10}$. According to Eq. (23), the shift of the average field at the $\mu^{+}$with respect to the applied field (measured by the frequency of the background signal, $\omega_{b}$ ) is given by

$$
\begin{aligned}
\left\langle\mathbf{B}_{\mu}\right\rangle-\mathbf{H}_{\mathrm{ext}}= & -\rho_{P} N_{P} \mathbf{M}_{P}+\rho_{P}\left\langle N_{H}\right\rangle \mathbf{M}_{P}+4 \pi \rho_{G}\langle\mathbf{M}(\theta)\rangle \\
& -\rho_{G}\left\langle N_{G}\right\rangle\langle\mathbf{M}(\theta)\rangle .
\end{aligned}
$$

Assuming that the average shape of individual grains can be approximated by a sphere we write $\left\langle N_{H}\right\rangle=\left\langle N_{G}\right\rangle=4 \pi / 3$ and further $\langle\mathbf{M}(\theta)\rangle=\mathbf{M}_{P}$ and 


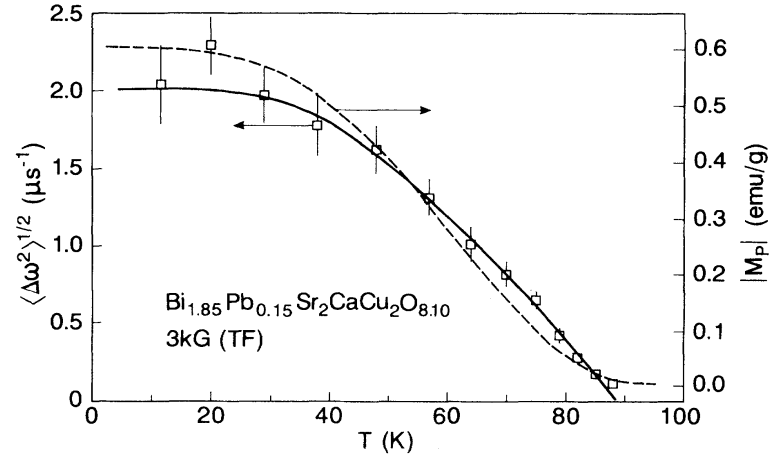

FIG. 11. Temperature dependence of $\left\langle\Delta \omega^{2}\right\rangle_{\text {eff }}$ [Eq. (33)] in $\mathrm{Bi}_{1.85} \mathrm{~Pb}_{0.15} \mathrm{Sr}_{2} \mathrm{CaCu}_{2} \mathrm{O}_{8.10}$. The solid line represents the BCS weak-coupling temperature dependence of $\lambda_{a b}^{2}(0) / \lambda_{a b}^{2}(T)$, the dashed line shows the temperature dependence of the fieldcooled sample magnetization $M_{P}$.

hence

$$
\left\langle B_{\mu}\right\rangle-B_{\mathrm{ext}} \cong\left[\left(\frac{4 \pi}{3}-N_{P}\right) \rho_{P}+\frac{8 \pi}{3} \rho_{G}\right] M_{P} .
$$

The frequency shift $\left(\langle\omega\rangle-\omega_{b}\right)=\gamma_{\mu}\left(\left\langle B_{\mu}\right\rangle-B_{\text {ext }}\right)$ should thus scale with the sample magnetization. This is indeed observed as can be seen from Fig. 12, which displays also the measured temperature dependence of $M_{P}(T)$ normalized to $\left\langle\omega_{\mu}\right\rangle-\omega_{b}$ at the lowest temperature point.

If the second moment $\left\langle\Delta \omega^{2}\right\rangle$ arises from the vortexlattice field distribution, it should be given by Eq. (19), i.e.,

$$
\left\langle\Delta \omega^{2}(T)\right\rangle_{\mathrm{eff}} \propto \frac{1}{\lambda_{a b}^{4}(T)} .
$$

The solid line in Fig. 11 was calculated assuming the temperature dependence of $\lambda(T)$ to follow the BCS predictions for weak coupling. It describes the data quite well. However, this must not be taken as evidence that it is indeed the vortex-lattice field distribution which determines primarily the $\mu^{+}$frequency distribution, as we will see later in more detail. The temperature dependencies

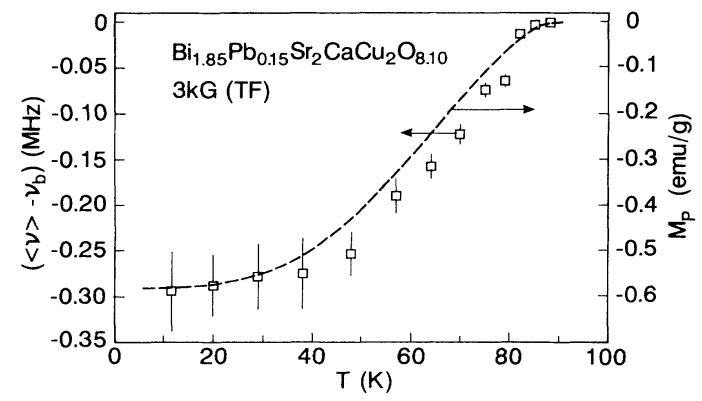

FIG. 12. Temperature dependence of $\left(\langle v\rangle-v_{b}\right)$ [see Eq. (34)] in $\mathrm{Bi}_{1.85} \mathrm{~Pb}_{0.15} \mathrm{Sr}_{2} \mathrm{CaCu}_{2} \mathrm{O}_{8.10}$. The dashed line shows again the temperature dependence of the field-cooled magnetization $M_{P}$. As can be seen $\left(\langle v\rangle-v_{b}\right)$ scales well with $M_{P}$ as expected $\left[v_{b}=\left(\gamma_{\mu} / 2 \pi\right) B_{\text {ext }}\right]$.

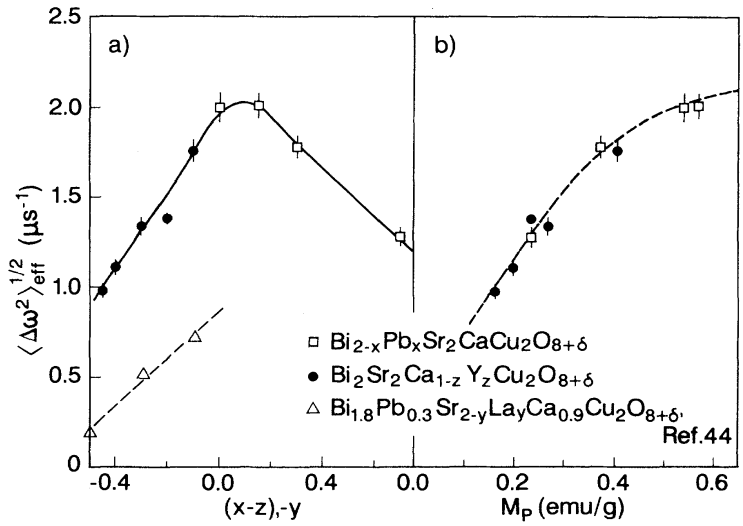

FIG. 13. Summary on the results for $\left\langle\Delta \omega^{2}\right\rangle_{\text {eff }}^{1 / 2}$ at $10 \mathrm{~K}$ for all samples, displayed in (a) as a function of $x-z$ and $-y$ and in (b) as a function of $M_{P}(10 \mathrm{~K})$. Included in (a) are the results (onecomponent $\sigma$ ) of Ref. 44.

seen in Figs. 11 and 12 are typical for all samples investigated. In particular, $\left(\langle\omega\rangle-\omega_{b}\right) / \gamma_{\mu}$ scales always well with the measured $M_{P}$ and the observed scaling factor $(\simeq 36)$ is closely reproduced by $\left(4 \pi / 3-N_{P}\right) \rho_{P}$ $+(8 \pi / 3) \rho_{G} \simeq 30$.

Finally, Fig. 13 summarizes the results on $\left(\Delta \omega^{2}\right)_{\text {eff }}^{1 / 2}$ at $T \simeq 10 \mathrm{~K}$ for all samples investigated. In Fig. 13(a), $\left\langle\Delta \omega^{2}\right\rangle_{\text {eff }}^{1 / 2}$ is plotted versus $x-z$ and in Fig. 13(b) versus the measured $M_{P}$ at $10 \mathrm{~K}$. From the latter, one may get the impression that $\left\langle\Delta \omega^{2}\right\rangle_{\text {eff }}^{1 / 2}$ could reflect, to a large extent, the bulk magnetization. It is, therefore, mandatory to take seriously into account possible broadening mechanisms which involve the magnetization of the samples.

\section{ANALYSIS OF RESULTS}

In this section we will concentrate exclusively on the $\sim 10 \mathrm{~K}$ results of all the investigated compounds with the aim of determining the London penetration depth proper. Our approach consists in a comparison of measured and simulated data, both by comparing real amplitude Fourier transforms and two-component fits. We begin by selecting a certain $\lambda_{a b}$ and, assuming $\lambda_{c} / \lambda_{a b}=55$, we calculate for 150 equally spaced values of $\theta$ in the interval $0^{\circ}-90^{\circ}$ the distributions $F_{\theta}(\omega)$. Next, in calculating the polycrystalline average $\left\langle\boldsymbol{F}_{\theta}(\omega)\right\rangle_{\text {poly }}$ the anisotropic magnetization, given by Eqs. (24), (26), or (27), is taken into account via Eq. (23) as described in Sec. III B. $M_{0}$ and $M_{1}$ in Eqs. (24), (26), and (27) were determined by identifying $\langle M(0)\rangle_{\text {poly }}$ with the measured magnetization at low temperatures, collected in Table $I$, and from the ratio $M\left(0^{\circ}\right) / M\left(90^{\circ}\right)=6.5$ taken from Ref. 32. Additional assumptions entering into this first step are as follows:

(i) The induction $\mathbf{B}$ and $\mathbf{B}_{\text {ext }}$ are parallel for all angles $\theta$. This is justified if $\mathbf{B}_{\text {ext }}>H_{c_{1}} \cdot{ }^{40,41}$

(ii) The flux lattice within an individual grain is absolutely regular, i.e., distortions due to pinning, etc., are ignored. We justify this with the following argument: if pinning or trapping is important it can be expected that it 
will become more pronounced with increasing $\mathrm{Pb}$ or $\mathrm{Y}$ content, leading to additional line broadening. ${ }^{25}$ The fact that $\left\langle\Delta \omega^{2}\right\rangle_{\text {eff }}$ rather decreases with rising $\mathrm{Y}$ or $\mathrm{Pb}$ content (see Fig. 13) suggests that flux-line disorder due to trapping is of minor influence.

In a second step the Gaussian distribution arising from the $\mathrm{Cu}$ nuclear dipoles is folded in, as well as the Gaussian distribution arising from the demagnetization factors $N_{G}$, as discussed in Sec. III C.

Next a background signal is added, accounting for the $\mu^{+}$stopping outside the sample. It is taken from fits to the experimental data at the lowest temperatures (see Sec. IV C). The thus obtained overall spectral distribution is then used to generate an artificial $\mu$ SR time spectrum by use of Eq. (21) and by taking into account the $\mu^{+}$decay and imposing statistical Gaussian noise. The length of this spectrum is adjusted to the length of the experimental histograms. This artificial spectrum can now be analyzed in the same way as the experimental ones. In particular, Fourier transforms $\widehat{P}(\omega)$ can be compared directly to each other, since the effect of the finite length of the histograms is now properly included. The artificial spectra in time space can also be fitted with a threecomponent expression [see Eqs. (31) and (32)] just as the experimental data.

The only really free variable in these calculations is the penetration depth $\lambda_{a b}$. All other parameters $\left(M_{0}, M_{1}, \sigma_{\mathrm{Cu}}, \Delta N_{G}\right)$ are taken from independent measurements. There is still a choice for the form of $M(\theta) . \lambda_{a b}$ is then varied until the generated and experimental Fourier transforms agree closely within their statistical noise (judging by eye) and, more qualitatively, until the maxima of the Fourier amplitudes (the latter quantity is particularly sensitive) and the parameters from the three-component fits of both the generated and the experimental time spectra agree within their statistical error bars. Likewise all three expressions for $M(\theta)$ are tried. As an example Fig. 14 shows "best" results for the compound $\mathrm{Bi}_{1.85} \mathrm{~Pb}_{0.15} \mathrm{Sr}_{2} \mathrm{CaCu}_{2} \mathrm{O}_{8.10}$ using all three expressions for $M(\theta)$. Depending on the choice of $M(\theta)$ the thus extracted $\lambda_{a b}$ varies between 1900 and $2500 \AA$. A closer inspection of the line shapes in Fig. 14 reveals that agreement with the data is least satisfactory for the choice of Eq. (26) for $M(\theta)$. The two other forms of $M(\theta)$ reproduce the data equally well in Fig. 14. However considering all compounds, the choice of Eq. (27) for $M(\theta)$ appears slightly better. [This is consistent with the data of Niskanen, ${ }^{32}$ which are well described by Eq. (27) but only poorly by Eq. (24) (see Fig. 4).] Although Eq. (27) cannot be justified theoretically the final analysis of all data was based on this expression. The results for $\lambda_{a b}$ are listed in Table II together with the hole concentration $p$ calculated from Eq. (3). The errors are estimates based on how much the input $\lambda_{a b}$ had to be changed in order to lead to differences between the fitted two-component parameters from the generated and measured spectra exceeding the sum of their error bars.

A surprisingly good description of the measured Fourier spectrum in Fig. 14 is also obtained by ignoring the magnetization altogether but allowing the ratio $\lambda_{c} / \lambda_{a b}$ and an additional Gaussian width $\widetilde{\sigma}_{a}$ due to some

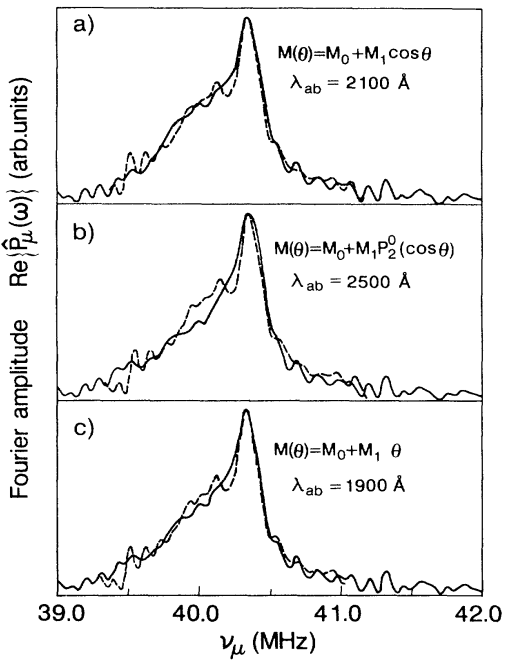

FIG. 14. Fourier spectrum of the $\mu \mathrm{SR}$ signal measured in $\mathrm{Bi}_{1.85} \mathrm{~Pb}_{0.15} \mathrm{Sr}_{2} \mathrm{CaCu}_{2} \mathrm{O}_{8.10}$ at $11.6 \mathrm{~K}$ after field cooling in $3 \mathrm{kG}$ (dashed lines) and best generated spectra assuming different expressions for $\boldsymbol{M}(\theta)$ (solid lines). See text for details.

unspecified broadening to become variables, leading to $\lambda_{a b}=1200 \AA, \lambda_{c} / \lambda_{a b}=7$, and $\widetilde{\sigma}_{a}=0.7 \mu \mathrm{s}^{-1}$. The increased $\widetilde{\sigma}_{a}$ may still arise in part from the distribution in demagnetization factors but could also point to a distortion of the flux-line lattice, as discussed in Sec. II. Indeed many $\mu \mathrm{SR}$ results on high- $T_{c}$ compounds have been analyzed just in this way (see, e.g., Ref. 42). However, generally for all compounds, $\lambda_{a b}$ and $\lambda_{c} / \lambda_{a b}$ had to be chosen considerably smaller in order to reproduce the data as compared to the former procedure. This widens the discrepancy, at least in compounds of the 2:2:1:2 family, with results obtained by other methods (Table III). Also $\lambda_{c} / \lambda_{a b}$ appears definitely too small in view of effective-mass ratio measurement. ${ }^{11,17}$ Given the fact that $M(\theta)$ is anisotropic we believe that our approach is basically well founded. Admitting a flux-line lattice distortion also in our treatment would shift $\lambda_{a b}$ to even larger values bringing it closer to results obtained by other techniques. We have not actually tried to analyze the data in this way, since an additional $\widetilde{\sigma}_{a}$ leads quickly to a more symmetric line shape in disagreement with the data.

Further checks concerned the influence of the ratios $\lambda_{c} / \lambda_{a b}$ and $M\left(0^{\circ}\right) / M\left(90^{\circ}\right)$ and the assumed width of the distribution of demagnetization factors, $\Delta N_{G}$. Increasing $\lambda_{c} / \lambda_{a b}$ from 55 to 1000 has a negligible influence on the spectral distributions. Changing $M\left(0^{\circ}\right) / M\left(90^{\circ}\right)$ from 6.5 to 55 changes the best value for $\lambda_{a b}$ by less than $10 \%$. Changing $\Delta N_{G}$ between zero and $4 \pi / 3$ produces only a change in the best $\lambda_{a b}$ of less than $3 \%$.

For further discussion we calculated from the obtained best $\lambda_{a b}$ values the vortex-lattice second moment $\left\langle\Delta \omega^{2}\right\rangle_{\text {vortex }} \propto 1 / \lambda_{a b}^{4}$ using Eq. (20). The dependence of $\left\langle\Delta \omega^{2}\right\rangle_{\text {vortex }}^{1 / 2}$ on the hole concentration $p$ [see Eq. (3) and Table II] is displayed in Fig. 15. It is seen that $\left\langle\Delta \omega^{2}\right\rangle_{\text {vortex }}^{1 / 2}$ rises linearly with $p$ for the $\mathrm{Y}$-doped compounds, while it is nearly independent of $p$ in the $\mathrm{Pb}$ doped compounds. Comparing this figure with Fig. 13 it 


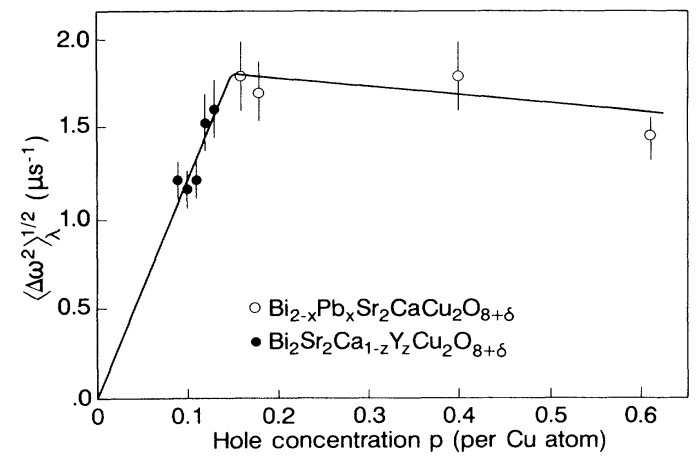

FIG. 15. Plot of the vortex-lattice second moment $\left\langle\Delta \omega^{2}\right\rangle_{\text {vortex }}^{1 / 2}$ versus the hole concentration $p$.

becomes clear that the decline of $\left\langle\Delta \omega^{2}\right\rangle_{\text {eff }}^{1 / 2}$ for rising $\mathrm{Pb}$ content is essentially a result of a reduced contribution from the anisotropic magnetization. If $\left\langle\Delta \omega^{2}\right\rangle_{\text {eff }}^{1 / 2}$ is plotted versus the hole concentration $p$ no smooth dependence for the Y-doped compounds does result.

\section{DISCUSSION}

Assuming that the extracted values for $\lambda_{a b}$ reflect the true London penetration depth [keeping in mind that the quoted values for $\lambda_{a b}$ (Table II) may have to be understood as lower limits] we try to understand the results on the basis of the London expression for the penetration depth [Eq. (1)], taking into account a correction related to the purity of the samples:

$$
\frac{1}{\lambda_{a b}^{2}}=\frac{4 \pi n_{s} e^{2}}{m_{a b} c^{2}} \frac{1}{1+\xi / l} .
$$

Here $n_{s}$ is the density of superconducting carriers, $m_{a b}$ is the effective mass in the basal plane, $\xi$ is the coherence length, and $l$ is the electron mean-free-path length. The factor $(1+\xi / l)^{-1}$ accounts for the purity of the sample via the free mean path.

It is possible that the dependence of $\lambda_{a b}$ on the $\mathrm{Pb}$ or $\mathrm{Y}$ content could reflect the dependence of $l$ on $\mathrm{Pb}$ or Y doping, which introduce a certain disorder. However, two observations seem to disfavor such an explanation: (i) $\xi$ is only of the order of $30 \AA$ or below and $l$ would have to be extremely short to influence $\lambda_{a b}$. Very short $l$ are not supported by resistivity data in the normal state. ${ }^{43}$ (ii) In the Y-doped compounds, $1 / \lambda_{a b}^{2}$ appears to scale with the total hole concentration $p$ (see Fig. 16) but not with the $\mathrm{Y}$ content $z$. In the $\mathrm{Pb}$-doped compounds $1 / \lambda_{a b}^{2}$ is essentially constant despite a considerable variation of the $\mathrm{Pb}$ content. Hence it seems that a possible dependence of $l$ on the $\mathrm{Pb}$ or $\mathrm{Y}$ concentration is not reflected in the data.

The other possibility then is to trace back the dependence of $\lambda_{a b}$ on the $\mathrm{Y}$ and $\mathrm{Pb}$ content to their effect on the ratio $n_{s} / m_{a b}$, where $n_{s}$ is given by the hole concentration. At least the results in the Y-doped compounds favor such an explanation. Figure 16 shows a plot of $n_{s}\left(m_{a b} / m_{0}\right)^{-1}$, calculated from Table II and Eq. (1), versus the hole concentration $p$ for the compounds

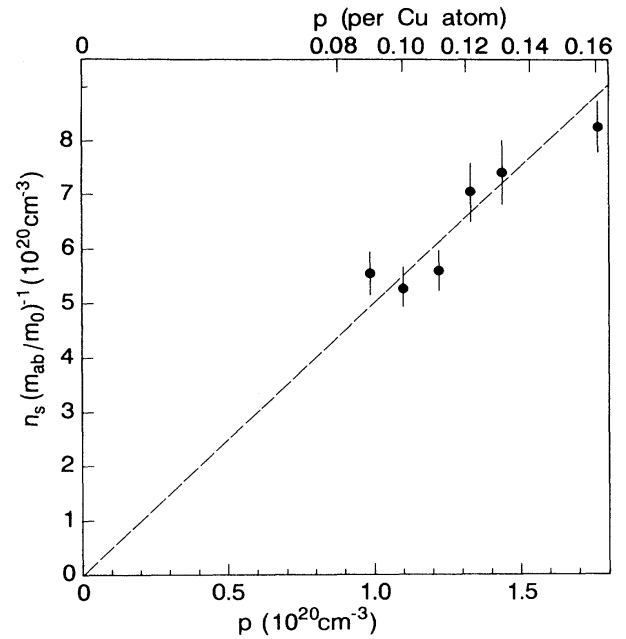

FIG. 16. Plot of $n_{s}\left(m_{a b} / m_{0}\right)^{-1}$ versus the hole concentration $p$ for the $\mathrm{Bi}_{2} \mathrm{Sr}_{2} \mathrm{Ca}_{1-x} \mathrm{Y}_{z} \mathrm{Cu}_{2} \mathrm{O}_{8+\delta}$ system. The data are compatible with a constant $m_{a b} / m_{0}$ which, if $n_{s}$ is identified with $p$, assumes a value $\simeq 5$.

$\mathrm{Bi}_{2} \mathrm{Sr}_{2} \mathrm{Ca}_{1-z} \mathrm{Y}_{z} \mathrm{Cu}_{2} \mathrm{O}_{8+\delta}(0 \leq z \leq 0.45)$. As can be seen the data are reasonably well represented by a straight line through the origin suggesting strongly that $\lambda_{a b}$ is determined by the actual two-dimensional hole concentration and that $m_{a b} / m_{0}$ is independent of doping.

The situation is different in the $\mathrm{Pb}$-doped compounds. $\lambda_{a b}$ hardly changes with the $\mathrm{Pb}$ content implying that the density of superconducting carriers is not affected by the holes introduced by replacing $\mathrm{Bi}^{3+}$ by $\mathrm{Pb}^{2+}$. This is consistent with the results of Hall-effect studies mentioned in the introduction, ${ }^{10}$ which show an almost constant Hall constant in the $\mathrm{Pb}$-doped compounds for hole concentrations exceeding 0.15 per $\mathrm{Cu}$ atom. Apparently the holes introduced by doping with $\mathrm{Pb}$ are not mobile and do contribute neither to the Hall conductivity nor the superconducting carrier density. The holes may be localized at $\mathrm{Pb}$ sites or alternatively at $\mathrm{O}$ sites. But note that the $\mathrm{Pb}$ content changes $T_{c}$ significantly.

Next we address the question of the scaling of $1 / \lambda_{a b}^{2}$ with $T_{c}$. Obviously this has to be restricted to the Ydoped compounds. Figure 17 shows a plot of $T_{c}$ versus $n_{s}\left(m_{a b} / m_{0}\right)^{-1}$ in comparison with a plot of $T_{c}$ versus the $\mathrm{Y}$ content $z$. As one can see $T_{c}$ decreases linearly and smoothly with increasing $z$, while no obvious relationship exists between $T_{c}$ and $n_{s}\left(m_{a b} / m_{0}\right)^{-1} \propto \lambda_{a b}^{-1}$. This has to be contrasted with Fig. 16, which shows that $\lambda_{a b}^{-2}$ is clearly linearly related to the hole concentration. We conclude that, at least for the Y-doped compounds, $T_{c}$ does not seem to scale with the hole concentration in clear contrast to $1 / \lambda_{a b}^{2}$. Rather, the smooth dependence of $T_{c}$ on $z$ could be suggestive of a nonelectronic effect pointing perhaps to a phonon-related mechanism. A similar behavior is seen in the only other systematic $\mu \mathrm{SR}$ study on members of the 2:2:1:2 family $\left[\mathrm{Bi}_{1.8} \mathrm{~Pb}_{0.3}\left(\mathrm{Sr}_{2-x} \mathrm{La}_{x}\right) \mathrm{Ca}_{0.9} \mathrm{Cu}_{2} \mathrm{O}_{8+\delta}\right)$ by Ansaldo et al. $\left.{ }^{44}\right]$ : $T_{c}$ declines linearly with the La content $x$ (see Fig. 18). Since $\delta$ was not determined the actual hole concentration 


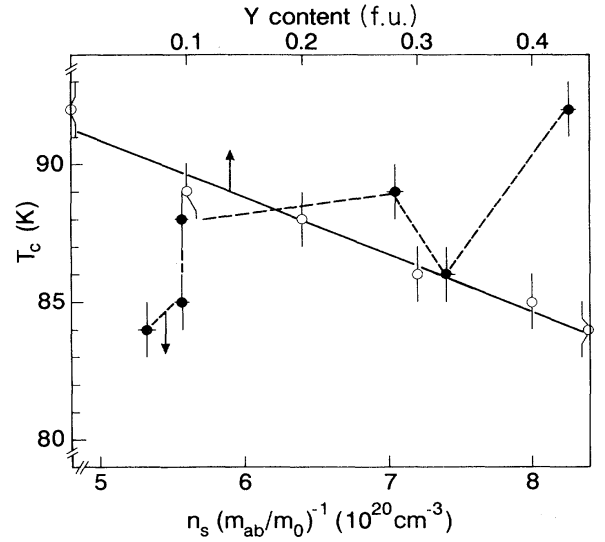

FIG. 17. Plot of $T_{c}$ vs the Y content $z$ and vs $n_{s}\left(m_{a b} / m_{0}\right)^{-1}$. $T_{c}$ decreases linearly with rising $z$, while no obvious correlation exists between $T_{c}$ and $n_{s}\left(m_{a b} / m_{0}\right)^{-1}$.

is not known in these compounds. One-component Gaussian fits yield $\sigma$ values which decrease with the La content very similarly to what is seen in the $\mathrm{Y}$-doped compounds (see Fig. 13). However, as Fig. 13 shows, the $\sigma$ values of Ref. 44 are generally smaller than the present results. Also $T_{c}$ drops much faster with increasing La content (see Fig. 18). The difference in behavior is difficult to understand, in part because the true hole concentration is not known in the La doped compounds. In any case, the reduced relaxation rates in these compounds imply smaller hole concentrations than in the Ydoped systems. The observation of simultaneously reduced hole concentrations and reduced $T_{c}$ (eventually $T_{c}$ becoming zero) is qualitatively consistent with the phase diagrams measured by Groen, de Leeuw, and Feiner, ${ }^{18}$ which shows a highly nonlinear correlation of $T_{c}$ and $p$. Figure 18 includes data from Ref. 18. It can be noticed that above $z=0.3, T_{c}$ decreases again linearly with $z$

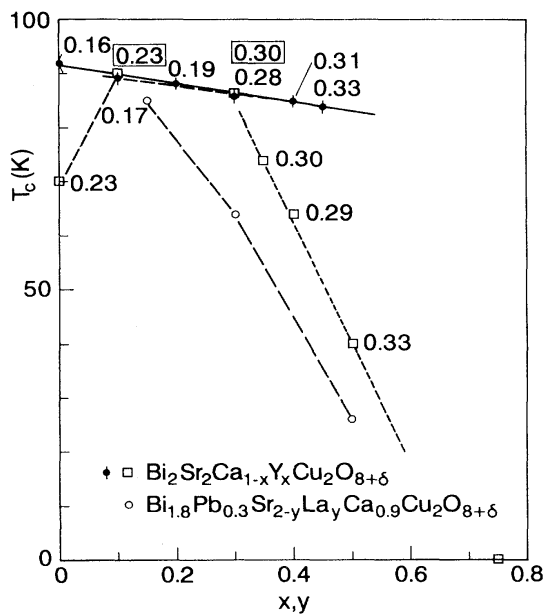

FIG. 18. Dependence of $T_{c}$ on $\mathrm{Y}$ and La doping in two different 2:2:1:2 systems. ( $\square$ from Ref. 18, $\bigcirc$ from Ref. 44, $\bullet$ present results). The values attached to the data points represent the oxygen excess $\delta$.

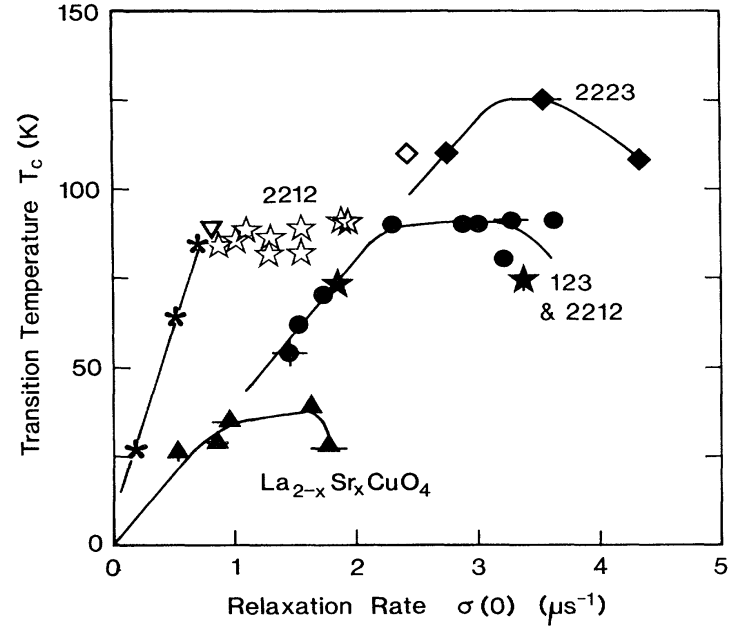

FIG. 19. Correlation between $T_{c}$ and the relaxation rate $\sigma$ (from one-component fits). All entries represented by filled symbols are taken from Refs. 4 and 5. The open stars refer to the present results. $\nabla$ was obtained in a $\mathrm{Bi}_{2} \mathrm{Sr}_{1.3} \mathrm{Ca}_{0.7} \mathrm{CuO}_{6.2}$ sample and $\diamond$ in a $\mathrm{Bi}_{1.83} \mathrm{~Pb}_{0.25} \mathrm{Sr}_{1.97} \mathrm{Ca}_{1.97} \mathrm{Cu}_{3.07} \mathrm{O}_{10+\delta}$ sample (M. Weber, Ref. 33). Included are also the results from Ref. 44.

(despite different $\delta$ ). It is also seen that, e.g., at $z=0.45-0.5$, our $T_{c}$ and the $T_{c}$ of Ref. 18 differ by almost a factor of 2 despite very close $\delta$ in contrast to the situation at $z=0.1$. Taken altogether, it seems that $1 / \lambda_{a b}^{2}$ is determined by the actual mobile hole concentration, while $T_{c}$ depends in a complicated, yet to be determined fashion and perhaps through different channels, reflecting different mechanisms, not only on the hole concentration and the oxygen content but separately also on the $\mathrm{Y}, \mathrm{Pb}$, or $\mathrm{La}$ doping. In conclusion we doubt that the universal scaling of $T_{c}$ with $1 / \lambda_{a b}^{2}$ for small hole concentrations, which is observed mainly in the $\mathrm{YBa}_{2} \mathrm{Cu}_{3} \mathrm{O}_{7-\delta}$ family, ${ }^{4,8,20}$ is also present in the 2:2:1:2 family. This will become particularly obvious if we include our results $(\sigma$ from one-component fits for the main signal) and the results from Ref. 44 in the diagram presented by Uemura et al. ${ }^{5}$ (see Fig. 19).

Finally we compare in Table III various results for $\lambda_{a b}$ in $\mathrm{Bi}_{2} \mathrm{Sr}_{2} \mathrm{CaCuO}_{8+\delta}$ obtained by different methods. Our value is significantly smaller than all the other ones (with the exception of the result from a torque measurement). ${ }^{17}$

TABLE III. Compilation of $\lambda_{a b}$ values obtained by different methods in $\mathrm{Bi}_{2} \mathrm{Sr}_{2} \mathrm{CaCu}_{2} \mathrm{O}_{8+\delta}$ (* same sample).

\begin{tabular}{lccc}
\hline & Method & $\lambda_{a b}(T \rightarrow 0 \mathrm{~K})[\AA]$ & \\
\hline $\mathrm{Bi}_{2} \mathrm{Sr}_{2} \mathrm{CaCu}_{2} \mathrm{O}_{8.16}^{*}$ & $\mu^{+} \mathrm{SR}$ & 1850 & This work \\
$\mathrm{Bi}_{2} \mathrm{Sr}_{2} \mathrm{Cu}_{2} \mathrm{O}_{8+\delta}$ & $M(H)$ & 3100 & Ref. 43 \\
$\mathrm{Bi}_{2} \mathrm{Sr}_{2} \mathrm{CaCu}_{2} \mathrm{O}_{8.16}^{*}$ & & 2900 & Ref. 48 \\
$\mathrm{Bi}_{2} \mathrm{Sr}_{2} \mathrm{CaCu}_{2} \mathrm{O}_{8+\delta}$ & & 3900 & Ref. 29 \\
$\mathrm{Bi}_{2} \mathrm{Sr}_{2} \mathrm{CaCu}_{2} \mathrm{O}_{8+\delta}$ & & 3000 & Ref. 49 \\
$\mathrm{Bi}_{2} \mathrm{Sr}_{2} \mathrm{CaCu}_{2} \mathrm{O}_{8+\delta}$ & $H_{c 1}$ & 3000 & Ref. 12 \\
$\mathrm{Bi}_{2} \mathrm{Sr}_{2} \mathrm{CaCu}_{2} \mathrm{O}_{8+\delta}$ & EPR & $<5000$ & Ref. 50 \\
$\mathrm{Bi}_{2} \mathrm{Sr}_{2} \mathrm{CaCu}_{2} \mathrm{O}_{8+\delta}$ & Torque & 1100 & Ref. 17 \\
$\mathrm{Bi}_{2} \mathrm{Sr}_{2} \mathrm{CaCu}_{2} \mathrm{O}_{y}$ & rf & 5000 & Ref. 51 \\
\hline \hline
\end{tabular}


As mentioned before this may be a result of underestimating the broadening contributions during field cooling in a polycrystalline sample. Interestingly measurements on single-crystalline material tend to yield generally smaller relaxation rates, ${ }^{45-47}$ even in the case that the external field is parallel to the $c$ direction. This may indeed be taken as evidence that the true $\lambda_{a b}$ is larger than what is generally extracted from $\mu \mathrm{SR}$ measurements on polycrystalline samples, including the present ones.

\section{SUMMARY}

We have presented low-temperature results from a systematic study of the width of the internal field distribution after field cooling through $T_{c}$ in the mixed state of high-temperature superconductors belonging to the 2:2:1:2 family: $\mathrm{Bi}_{2} \mathrm{Sr}_{2} \mathrm{Ca}_{1-z} \mathrm{Y}_{z} \mathrm{Cu}_{2} \mathrm{O}_{8+\delta}$, changing the concentration of the electron donor $\mathrm{Y}$, and $\mathrm{Bi}_{2-x} \mathrm{~Pb}_{x} \mathrm{Sr}_{2} \mathrm{CaCu}_{2} \mathrm{O}_{8+\delta}$ changing the concentration of the hole donor $\mathrm{Pb}$. In trying to fully understand and exploit the observed line shapes and amplitudes of the Fourier transformed $\mu \mathrm{SR}$ spectra, we include in the analysis not only the spectral field distribution expected to arise from an anisotropic superconductor in the mixed or Abrikosov state, which is determined by the London penetration depth, but also the distribution arising from the pronounced anisotropic magnetization in the polycrystalline samples. Other contributions considered and taken into account include the nuclear dipole fields originating from the $\mathrm{Cu}$ nuclear magnetic moments and the distribution in demagnetization factors associated with varying grain shapes present in the investigated coldpressed samples. We came to the conclusion that texture, macroscopic field gradients in the grains due to Bean's critical state model, and disorder in the flux-line lattice can be ignored, at least as far as the measured $\mu$ SR line shape is concerned. Flux melting and glassy features should be absent, since the reported results were all obtained at low temperatures $(\sim 10 \mathrm{~K})$ and not too large applied fields $(3 \mathrm{kG})$, i.e., well below the phase boundary to the molten flux-line state. We have obtained best values of the penetration depth $\lambda_{a b}$ (assuming $\lambda_{c} / \lambda_{a b} \geq 55$, deduced from torque magnetometry) by requiring the shape and amplitudes of Fourier transformed measured and generated spectra to coincide within their error bars. We find that in the Y-doped samples $1 / \lambda_{a b}^{2} \propto n_{s} / m_{a b}$ scales well with the hole concentration following from the measured oxygen excess and the $\mathrm{Y}$ content, while $T_{c}$ in these compounds scales very well only with the $Y$ content. In contrast, in the $\mathrm{Pb}$-doped samples $1 / \lambda_{a b}^{2}$ turns out to be independent of doping concentration, in essentially good agreement with Hall-effect measurements, implying that the holes provided by the $\mathrm{Pb}^{+2}$ ions are not mobile and do not contribute to the superconducting carrier density and neither to the Hall conductivity. Our results from the 2:2:1:2 family are in disagreement with the universal $T_{c}$ versus $\sigma$ curve put forward by Uemura et al. ${ }^{5}$ and suggest instead that $T_{c}$ is a more complicated function of not only the actual hole concentration but separately also of the $\mathrm{Y}$ or $\mathrm{Pb}$ content. In view of this it would be very interesting to study the dependence of $T_{c}$ on the oxygen excess and the doping concentrations separately in much more detail.

\section{ACKNOWLEDGMENTS}

We are extremely grateful to Karina Niskanen (Department of Technology, University of Uppsala) for providing us with unpublished results on her study of the anisotropy of field-cooled magnetization. The Russian members of this collaboration acknowledge the continuous encouragement and support of the late Professor I. I. Gurevich and Dr. B. A. Nikolsky. The technical assistance of I. A. Gaganov is greatly appreciated. We thank Professor H. R. Ott for his keen interest in this work and for many helpful and stimulating discussions. Part of this work was supported by the Scientific Council of the Russian Federation on high- $T_{c}$ problems (Grant No. 90559 of the State Programme "High-Temperature Superconductivity").
${ }^{1}$ See, e.g., D. Saint-James, G. Sarma, and E. J. Thomas, Type-II Superconductivity (Pergamon, Oxford, 1969).

${ }^{2}$ S. L. Thiemann, Z. Radovic, and V. G. Kogan, Phys. Rev. B 39, 11406 (1989).

${ }^{3}$ A. Schenck, Muon-Spin-Rotation Spectroscopy (Hilger, Bristol, 1985).

${ }^{4}$ Y. J. Uemura, V. J. Emery, A. R. Moodenbaugh, M. Suenaga, D. C. Johnston, A. J. Jacobson, J. T. Lewandowski, J. H. Brewer, R. F. Kiefl, S. R. Kreitzman, G. M. Luke, T. Riseman, C. E. Stronach, W. J. Kossler, J. R. Kempton, X. H. Yu, D. Opie, and H. E. Schone, Phys. Rev. B 38, 909 (1988).

${ }^{5}$ Y. J. Uemura, L. P. Le, G. M. Luke, B. J. Sternlieb, W. D. Wu, J. H. Brewer, T. M. Riseman, C. L. Seaman, M. B. Maple, M. Ishikawa, D. G. Hinks, J. D. Jorgensen, G. Saito, and H. Yamocki, Phys. Rev. Lett. 66, 2665 (1991).

${ }^{6}$ T. Schneider, Z. Phys. B 88, 249 (1992).

${ }^{7}$ C. Parucchini, L. Romanó, and S. Bellini, Physica C 192, 443
(1992).

${ }^{8}$ H. Glückler, Ch. Niedermayer, G. Novitzke, E. Recknagel, J. Erxmeyer, A. Weidinger, and J. I. Budnick, Europhys. Lett. 15, 355 (1991).

${ }^{9}$ C. L. Seaman, J. J. Neumeier, M. B. Maple, L. P. Le, G. M. Luke, B. J. Sternlieb, Y. J. Uemura, J. H. Brewer, R. Kadono, R. F. Kiefl, S. R. Kreitzman, and T. M. Riseman, Phys. Rev. B 42, 6801 (1990).

${ }^{10}$ H. Maeda, M. Hase, I. Tsukada, K. Noda, S. Takebayashi, and K. Uchinokara, Phys. Rev. B 41, 6418 (1990).

${ }^{11}$ D. E. Farell, S. Bonheam, J. Foster, Y. C. Chang, P. Z. Jian, K. G. Vandervoort, D. J. Lam, and V. G. Kogan, Phys. Rev. Lett. 63, 782 (1989).

${ }^{12}$ S. Gygax, W. Xing, O. Rajora, and A. Curzon, Physica C 162-164, 1551 (1989).

${ }^{13}$ T. T. M. Palstra, B. Batlogg, L. F. Schneemeyer, R. B. van Dover, and J. V. Waszczak, Phys. Rev. 38, 5102 (1988). 
${ }^{14}$ O. Laborde, P. Monceau, M. Potel, P. Gaugeon, J. Padiou, J. C. Levet, and H. Noel, Solid State Commun. 67, 609 (1988).

15J. J. Lin, E. L. Benitez, S. J. Poon, M. A. Subramanian, J. Gopalakrishnan, and A. W. Sleight, Phys. Rev. 38, 5095 (1988).

${ }^{16}$ D. E. Farrell, R. G. Beck, M. F. Booth, C. J. Allen, E. D. Bukowski, and D. M. Ginsberg, Phys. Rev. B 42, 6758 (1990).

${ }^{17}$ J. C. Martinez, S. H. Brongersma, A. Koshelev, B. Ivlev, P. H. Kes, R. P. Griessen, D. G. de Groot, Z. Tarnavsky, and A. A. Menovsky, Phys. Rev. Lett. 69, 2276 (1992).

${ }^{18}$ W. A. Groen, D. M. de Leeuw, and L. F. Feiner, Physica C 165, 55 (1990).

${ }^{19}$ V. Dallacasa and R. Feduzi, Phys. Lett. A 170, 153 (1992).

${ }^{20}$ B. Pümpin, H. Keller, W. Kündig, I. M. Savić, J. W. Schneider, H. Simmler, P. Zimmerman, E. Kaldis, S. Rusiecki, and C. Rossel, Hyperfine Interact. 63, 25 (1990).

${ }^{21}$ E. H. Brandt, Phys. Rev. B 937, 2349 (1988).

${ }^{22}$ P. Pincus, A. C. Gossard, V. Jaccarino, and J. H. Wernick, Phys. Rev. Lett. 13, 21 (1964).

${ }^{23}$ W. Barford and J. M. F. Gunn, Physica C 156, 515 (1988).

${ }^{24}$ V. I. Fesenko, V. N. Gorbunov, and V. P. Smilga, Physica C 176, 551 (1991).

${ }^{25}$ E. H. Brandt, J. Low Temp. Phys. 73, 355 (1988).

${ }^{26}$ A. M. Portis (unpublished).

${ }^{27}$ See, e.g., B. Goss Levi, Phys. Today 45, (10), 17 (1992).

${ }^{28}$ A. Abragam, The Principles of Nuclear Magnetism (Clarendon, Oxford, 1970).

${ }^{29}$ See, e.g., W. Kritscha, F. M. Sauerzopf, H. W. Weber, G. W. Crawtree, Y. C. Chang, and P. J. Jian, Physica C 179, 59 (1991).

${ }^{30}$ A. Schenck, Physica C 153-155, 1127 (1988); P. Birrer, F. N. Gygax, B. Hettich, B. Hitti, E. Lippelt, H. Maletta, A. Schenck, and M. Weber, ibid. 158, 230 (1989).

${ }^{31}$ I. Felner (private communication).

${ }^{32} \mathrm{~K}$. Niskanen (private communication).

${ }^{33}$ A. Schenck et al., Hyperfine Interact. 63, 227 (1990); M. Weber, Ph.D. thesis, ETH, Zürich, 1991.

${ }^{34}$ D. R. Harshman, A. T. Fiory, and R. J. Cava, Phys. Rev. Lett. 66, 3313 (1991).

${ }^{35}$ D. R. Harshman, E. H. Brandt, A. T. Fiory, M. Inui, D. B. Mitzi, L. F. Schneemeyer, and J. V. Waszczak, Phys. Rev. B 47, 2905 (1993).

36J. Bock, S. Elschner, and E. Preisler, in Advances in Superconductivity III, Proceedings of the ISS ' 90 , Sendai, edited by K.
Kajimura and H. Hayakawa (Springer-Verlag, Tokyo, 1991), p. 797.

37J. Bock, E. Preisler, and S. Elschner, in Modern Superconductivity, Proceedings of the ICMAS '90, Grenoble, edited by R. Tournier and R. Suryanarayanan (IITT, Gournay sur Marne, 1990), p. 13.

${ }^{38}$ L. W. Kirenski, Magnetismus (Teubner, Leipzig, 1969).

${ }^{39}$ V. H. Dodokhov, V. N. Duginov, I. A. Gaganov, V. G. Grebinnik, S. Kapusta, A. B. Lazarev, V. G. Olshevsky, V. Yu. Pomjakushin, V. S. Roganov, S. N. Shilov, V. A. Zhukov, V. G. Zinov, I. I. Gurevich, B. F. Kirillov, E. P. Krasnoperov, B. A. Nikolsky, A. V. Pirogov, A. N. Ponomarev, V. G. Storchak, V. A. Suetin, S. Safrata, and J. Sebek, Hyperfine Interact. 65, 1167 (1990).

${ }^{40}$ L. J. Campbell, M. M. Doria, and V. G. Kogan, Phys. Rev. 38, 2439 (1988).

${ }^{41}$ D. E. Farell, C. M. Williams, S. A. Wolf, N. P. Bansal, and V. G. Kogan, Phys. Rev. Lett. 61, 2805 (1988).

${ }^{42}$ B. Pümpin, H. Keller, W. Kündig, W. Odermatt, I. M. Savić, J. Schneider, K. Kaldis, S. Rusiecki, Y. Maeno, and C. Rossel, Phys. Rev. B 42, 8019 (1990).

${ }^{43}$ A. Schilling, F. Hulliger, and H. R. Ott, Z. Phys. B 82, 9 (1991).

${ }^{44}$ E. J. Ansaldo, J. Boyle, C. Niedermayer, H. Glückler, J. L. Tallon, and A. Mawdsley, Physica C 185-189, 747 (1991).

${ }^{45}$ B. J. Sternlieb, L. P. Le, G. M. Luke, Y. J. Uemura, J. H. Brewer, J. F. Carolan, P. Dosanjh, W. N. Hardy, R. Kadono, R. F. Kiefl, S. R. Kreitzman, T. M. Riseman, P. W. Schleger, M. R. Davis, C. E. Stronach, Y. Hidaka, T. Murakami, J. Gopalakrishnan, A. W. Sleight, M. Subramanian, and A. R. Strzelecki, Physica C 162-164, 679 (1989).

${ }^{46}$ E. M. Forgan, S. L. Lee, S. Sutton, J. S. Abell, S. F. J. Cox, C. A. Scott, H. Keller, B. Pümpin, J. W. Schneider, H. Simmler, P. Zimmermann, and I. M. Savić, Hyperfine Interact. 63, 71 (1990).

${ }^{47} \mathrm{H}$. Maletta et al. (private communication).

${ }^{48} \mathrm{~A}$. Schilling (private communication).

${ }^{49}$ S. Mitra, J. H. Cho, W. C. Lee, D. C. Johnston, and V. G. Kogan, Phys. Rev. B 40, 2674 (1989).

${ }^{50}$ M. Wahlen, M. Rübsam, O. Dobbert, and K.-P. Dinse, Physica C 185-189, 1797 (1991).

${ }^{51}$ A. Maeda, T. Shibauchi, N. Kondon, K. Uchinokura, and M. Kabayashi, Phys. Rev. B 46, 14234 (1992). 